FICHA CATALOGRÁFICA

Preparada pelo Serviço de Biblioteca e Informação do Instituto de Física da Universidade de São Paulo

Andrade, Débora Machado

Desigualdade de Jensen aplicada à probabilidade de fusão nuclear - São Paulo, 2009.

Dissertação (Mestrado) - Universidade de São Paulo. Instituto de Física, Departamento de Física Matemática

Orientador: Prof. Dr. Mahir Saleh Hussein

Área de Concentração: Física Nuclear

Unitermos: 1. Física Nuclear; 2. Reações Nucleares;

3. Fusão Nuclear; 4. Análise Matemática;

5. Desigualdades 
Universidade de São Paulo

Instituto de Física

\title{
Desigualdade de Jensen Aplicada à Probabilidade de Fusão Nuclear
}

\author{
Débora Machado Andrade
}

Orientador: Prof. Dr. Mahir Saleh Hussein

Banca examinadora:

(IF-USP) Mahir Saleh Hussein (IF-USP) Luiz Carlos Chamon

(ITA) Manuel Máximo Bastos

Malheiro de Oliveira
Dissertação de mestrado apresentada ao Instituto de Física para a obtenção do título de Mestre em Ciências

São Paulo 
Dedicada a Arsen Melikyan 


\section{Agradecimentos}

Ao professor Mahir Saleh Hussein, por ter sido meu orientador e exemplo de tantas coisas na vida. Por ter sido um verdadeiro mestre e ter me dado a honra de ser sua aluna.

Aos professores Emerson Passos, João Carlos Barata, Josif Frenkel e Ashok Das, por serem excelentes professores e físicos. Por inspirarem a busca pela ciência e ensino de qualidade.

A Amélia Ferrari e a Simone Shinomiya por serem as melhores secretárias do mundo.

A Wei Liang Qian, Rone Andrade, Ruben Pampa e Alexandr Pinzul, pela amizade e coleguismo que fizeram meu dia-a-dia no Instituto de Física ser sempre bastante agradável.

A Arsen Melikyan, por ter me encorajado e inspirado incansavelmente na física.

Aos amigos Danilo Dias, Danilo Nunes, Fabrício Resende e Marco Antônio Sampaio, por terem partilhado comigo toda esta experiência de estudar na USP. Por terem se tornado parte da minha família.

Por fim, aos meus pais, sem os quais nada faria sentido. Espero algum dia poder tornar concretas as idéias nobres e generosas que eles me inspiram. 


\section{Resumo}

Discutimos o problema de tunelamento quântico em sistemas físicos com muitos graus de liberdade. Aplicamos a desigualdade de Jensen ao cálculo analítico semi-clássico da probabilidade de fusão entre dois núcleos, onde consideramos graus de liberdade intrínsecos. Utilizamos diferentes barreiras de potencial de tunelamento e trabalhamos analiticamente com cada uma delas. Provamos matematicamente então a validade de uma desigualdade de caráter geral que relaciona a probabilidade de tunelamento de um sistema com vários graus de liberdade com a probabilidade de tunelamento do mesmo sistema aproximado para um modelo unidimensional, ou seja, com todos os graus de liberdade, exceto o radial, sendo tomados em média. Tal desigualdade já é conhecida empiricamente, através de cálculo numérico para diferentes modelagens, e tem particular relevância no problema da fusão de íons pesados a energias próximas da barreira de potencial. Mostramos que uma desigualdade envolvendo reflexão sobre a barreira de potencial, derivada por R. Johnson e C. Goebel, e utilizada para estimar o efeito de breakup no espalhamento elástico de núcleos halo, é uma consequência imediata da desigualdade de Jensen. Uma generalização das idéias contidas no referido trabalho de Johnson e Goebel, possibilitada pela aplicação da desigualdade de Jensen, enriquece a compreensão acerca de limites superiores e inferiores para a probabilidade de tunelamento quântico em sistemas com vários graus de liberdade. 


\begin{abstract}
We discuss the quantum tunneling problem in physical systems involving many degrees of freedom. We apply the Jensen inequality to the semi-classical analytical probability of fusion between two nuclei, where we considered intrinsic degrees of freedom. We employed different tunneling potential barriers and analytically worked on each of them. We have mathematically proven then the validity of a general inequality which relates the tunneling probability for a sub-system of the many-degrees-of-freedom system when compared to the sub-system alone (with the coupling to the reservoir being averaged). Such inequality is already empirically well-known through numerical calculations for different models, and has a particular relevance in the problem of heavy ion fusion at sub-barrier energies. We have shown that an inequality derived by R. Johnson and C. Goebel, which involves the reflection over a potential barrier and was used to estimate the breakup effect on the elastic scattering of halo nuclei, is but an immediate consequence of the Jensen inequality. A generalization of the ideas contained in the refered work of Johnson and Goebel, which was made possible by using the Jensen inequality, enriches the comprehension towards upper and lower boundaries for tunneling probabilities in systems with many degrees of freedom.
\end{abstract}




\section{Sumário}

1 Introdução 1

2 Tunelamento em Um Grau de Liberdade 4

3 Tunelamento em Sistemas Complexos e Efeito do Meio Externo 12

4 Desigualdade de Jensen para Probabilidades de Tunelamento 18

4.1 Desigualdade de Jensen Aplicada à Análise do Efeito de Breakup no Espalhamento Elástico de Núcleos Halo . . . . . . . . . . . . . . . . . . . . . . . . . . . . . 22

4.2 Desigualdade de Jensen Aplicada à Probabilidade de Tunelamento Através de Barreira Retangular . . . . . . . . . . . . . . . . . . . . . . . . . 24

4.3 Desigualdade de Jensen Aplicada à Probabilidade de Tunelamento Através de Barreira de Potencial Isocrônico . . . . . . . . . . . . . . . . . . . . . 25

4.4 Desigualdade de Jensen Aplicada à Probabilidade de Tunelamento Através de Barreira de Potencial Coulombiano . . . . . . . . . . . . . . . . . . . . . 30

4.5 Desigualdade de Jensen Aplicada à Probabilidade de Tunelamento Através de Barreira de Potenciais Nuclear, Coulombiano e Centrífugo Combinados . . . . . . . . 32

4.5.1 Desigualdades Para Fusão em Baixas Energias . . . . . . . . . . . . . 32

4.5.2 Desigualdades Para Todo o Espectro de Energia . . . . . . . . . . . . . . 37 
SUMÁRIO

4.6 Desigualdades Envolvendo um Fator de Forma Variante . . . . . . . . . . . . . . . 43

5 Conclusões $\quad 46$

6 APÊNDICE 49

Referências Bibliográficas $\quad 52$ 


\section{CAPÍtulo 1}

\section{Introdução}

Em sistemas quânticos complexos com muitos graus de liberdade operacionais, usualmente é verificada uma grande disparidade entre a probabilidade de tunelamento quântico observada e a prevista pelo modelo de penetração em barreira unidimensional simples. Experimentos de fusão de núcleos a energias pouco abaixo da barreira de potencial [1-5] mostraram claramente um grande aumento da probabilidade de tunelamento quando comparada à esperada pelo modelo de barreira unidimensional simples. O problema geral do tunelamento em barreira na presença de um meio externo foi abordado por Caldeira e Leggett [6-8] em conexão com o tunelamento do fluxo capturado em

um dispositivo supercondutor de interferência (SQUID), utilizando o funcional de influência de Feynman (FIF) $[9,10]$. Concomitantemente, muitos artigos teóricos importantes foram publicados abordando a questão da fusão nuclear [11-13]. Nesses trabalhos, esforços foram feitos para se obter estimativas semi-quantitativas, embora importantes, dos efeitos dos graus de liberdade do meio externo na dinâmica de tunelamento do sub-sistema de interesse. Por outro lado, cálculos numéricos mais detalhados baseados na descrição de canais acoplados da fusão sub-barreira [14-18] tentam dar uma descrição quantitativa restringindo as dimensões do reservoir (o número de canais fortemente acoplados ao canal de entrada). Seria um complemento útil para a discussão acima encontrar desigualdades gerais que envolvessem a probabilidade de tunelamento para um sub-sistema do sistema de muitos graus de liberdade e a probabilidade de tunelamento do sub-sistema isolado onde 
os graus de liberdade intrínsecos são tomados em média. Este é o principal objetivo deste trabalho. Para tanto, nos valemos de um teorema em análise matemática conhecido como teorema de Jensen. A desigualdade de Jensen, enunciada pela primeira vez pelo matemático dinamarquês J.L. Jensen no final do século XIX, pode ser enunciada concisamente da seguinte forma: "a transformação convexa da média de uma distribuição é menor ou igual que a média dos valores assumidos por tal transformação convexa daquela distribuição". Definições precisas e específicas desta desigualdade, a qual pode ser enunciada de várias formas, a depender da complexidade das transformações e distribuições envolvidas, serão dadas posteriormente neste trabalho.

A desigualdade de Jensen, sendo uma desigualdade fundamental da análise matemática, foi demonstrada através de argumentos baseados na teoria termodinâmica por A.R. Plastino, A. Plastino e G.H. Miller, num artigo denominado "Thermodynamic paths to Jensen's inequality" [19]. Nesse artigo, os autores verificam empiricamente a desigualdade de Jensen através de experiências hipotéticas de nivelação isotérmica de um fluido incompressível num sistema de vasos comunicantes, e também através da equilibração térmica de um sistema de muitos corpos.

A desigualdade de Jensen parece ter sido primeiramente aplicada na física estatística por R. Peierls [20] em 1938, visando à obtenção da seguinte desigualdade envolvendo a função de partição canônica $Z(\beta)$ :

$$
Z(\beta)=\operatorname{Tr}[\exp [-\beta H]] \geqslant \exp [-\beta \operatorname{Tr}[H]]
$$

Também R. Feynman [10] utilizou a desigualdade de Jensen em seu livro "Quantum Mechanics and Path Integrals", para enunciar o seguinte princípio variacional:

$$
F_{0} \leqslant F_{0}^{\prime}-\frac{1}{\beta}\left\langle S-S^{\prime}\right\rangle
$$

em que $F_{0}$ é a energia livre do sistema e $S$ é o análogo quântico da ação clássica correspondente àquela energia. Essa desigualdade mostra que se calcularmos $F_{0}^{\prime}-\frac{1}{\beta}\left\langle S-S^{\prime}\right\rangle$ para várias "ações" $\mathrm{S}^{\prime}$, então o cálculo que fornecer o menor valor estará o mais próximo possível da energia livre real $F_{0}$. 
Tendo esta desigualdade uma ampla gama de aplicações, recentemente tem sido utilizada em diversos campos da física aplicada, de processos estocásticos na biofísica à teoria de informação. Com efeito, como foi pontuado por J. Ruel e M. Ayres em seu artigo intitulado “Jensen's inequality predicts effects of environmental variations" [21], a desigualdade de Jensen tem relevância em todo campo da biologia que inclui processos não lineares. Por envolver comparações entre médias estatísticas, a desigualdade de Jensen tem larga aplicação em todas as ciências em que um tratamento estatístico das variáveis a serem consideradas se faz necessário.

Esta dissertação está organizada da seguinte forma: no capítulo 2 fazemos uma revisão sobre o tunelamento em um grau de liberdade; no capítulo 3 é discutido brevemente o problema do tunelamento em sistemas com vários graus de liberdade; no capítulo 4 abordamos o problema do efeito do meio externo, i.e. do reservoir, em probabilidades de tunelamento; o capítulo 5, onde aplicamos a desigualdade de Jensen a probabilidades de tunelamento e interpretamos os resultados, constitui o âmago desta dissertação, sendo totalmente original; no capítulo 6 é feita a conclusão deste trabalho. 


\section{Tunelamento em Um Grau de Liberdade}

Quando projetada sobre ondas parciais, a hamiltoniana de energia cinética no espaço de configuração se torna uma soma de sua parte diferencial radial mais um termo de potencial repulsivo centrífugo,

$$
-\frac{\hbar^{2}}{2 \mu} \nabla^{2}=-\frac{\hbar^{2}}{2 \mu} \frac{1}{r^{2}} \frac{d}{d r}\left(r^{2} \frac{d}{d r}\right)+\frac{\hbar^{2} l(l+1)}{2 \mu r^{2}}
$$

O termo centrífugo é então combinado com o potencial de tunelamento $V_{t}(r)$ para definir o potencial efetivo, dependente do número quântico $l$ da onda parcial correspondente, dado por

$$
V_{l}(r)=V_{t}(r)+\frac{\hbar^{2} l(l+1)}{2 \mu r^{2}}
$$

Utilizando a formulação de [22] para a probabilidade de transmissão abaixo da barreira, que garante uma transmissão de 1/2 no topo de uma barreira simétrica [23], e leva em consideração reflexões múltiplas de todas as ordens dentro da barreira de tunelamento, se a aproximação uniforme for usada numa fomulação do tunelamento através de integrais de trajetória [12], a probabilidade de tunelamento associada ao sub-sistema isolado tem a forma

$$
T_{l}\left[E, V_{l}(r)\right]=\frac{1}{1+\exp \left(g_{l}\left[E, V_{l}(r)\right]\right)}
$$


onde a ação $g_{l}\left[E, V_{l}(r)\right]$ é dada por

$$
g_{l}\left[E, V_{l}(r)\right]=\sqrt{\frac{8 \mu}{\hbar^{2}}} \int_{r_{1}(l, E)}^{r_{2}(l, E)} d r \sqrt{V_{l}(r)-E}
$$

e onde $r_{1}(l, E)$ e $r_{2}(l, E)$ são os pontos de retorno clássicos.

Uma maneira de obter uma forma fechada para a probabilidade de tunelamento acima é seguir o procedimento de Hill-Wheeler [24], que consiste em aproximar o potencial efetivo de tunelamento por uma parábola invertida, expandindo o potencial efetivo em torno da coordenada $R_{l}$ onde o potencial assume seu valor máximo, e mantendo apenas os termos de ordem mais baixa (quadrática). Com efeito, expandindo o potencial $V_{l}(r)$ em torno de $R_{l}$, obtemos:

$$
V_{l}(r)=V_{l}\left(R_{l}\right)+\left(r-R_{l}\right)\left[\frac{d V_{l}(r)}{d r}\right]_{r=R_{l}}+\frac{1}{2}\left(r-R_{l}\right)^{2}\left[\frac{d^{2} V_{l}(r)}{d r^{2}}\right]_{r=R_{l}}+O\left(r^{3}\right)
$$

Ora, como o ponto $R_{l}$ representa a coordenada do ponto de máximo da função, temos que $\left[\frac{d V_{l}(r)}{d r}\right]_{r=R_{l}}=$ 0 e $\left[\frac{d^{2} V_{l}(r)}{d r^{2}}\right]_{r=R_{l}}<0$. Descartando os termos de ordem 3 ou superior em $r$, chegamos à aproximação de Hill-Wheeler para a barreira de potencial de tunelamento:

$$
V_{H W_{l}}(r) \equiv V_{l}\left(R_{l}\right)-\frac{1}{2} \mu \omega_{l}^{2}\left(r-R_{l}\right)^{2}
$$

onde $\omega_{l}$ é proporcional à segunda derivada de $V_{l}(r)$ calculada no ponto $R_{l}$ :

$$
\omega_{l}=\sqrt{\frac{1}{\mu}\left|\frac{d^{2} V_{l}(r)}{d r^{2}}\right|_{r=R_{l}}}
$$

A partir da aproximação parabólica para o potencial, a ação $g_{l}\left[E, V_{l}(r)\right]$ tem a forma

$$
g_{l}\left[E, V_{l}(r)\right]=\sqrt{\frac{8 \mu}{\hbar^{2}}} \int_{r_{1}(l, E)}^{r_{2}(l, E)} d r \sqrt{V_{l}\left(R_{l}\right)-\frac{1}{2} \mu \omega_{l}^{2}\left(r-R_{l}\right)^{2}-E}
$$

a qual, após algumas mudanças de variáveis, pode ser reescrita na forma

$$
g_{l}\left[E, V_{l}(r)\right]=\frac{4}{\hbar \omega_{l}}\left(B_{l}-E\right) \int_{-\frac{\pi}{2}}^{\frac{\pi}{2}} d \theta(\cos \theta)^{2}
$$

podendo, então, ser calculada de forma fechada: 


$$
g(l, E)=\frac{2 \pi}{\hbar \omega_{l}}\left(B_{l}-E\right)
$$

onde $B_{l}=V_{l}\left(R_{l}\right)$.

Em aplicações à fissão e fusão nuclear, a aproximação acima para a ação permite o cálculo de observáveis de forma fechada.

Em fissão nuclear ou no decaimento de partículas muito carregadas, a taxa temporal de ocorrência dos eventos é calculada multiplicando a transmissão $l=0$ pelo número do fator de assaltos, ou seja, o número de vezes por segundo que o fragmento bate na barreira pelo lado de dentro antes de escapar:

$$
R=P_{\text {pre }}\left(\frac{v}{2 R}\right) \frac{1}{1+\exp \left[\frac{2 \pi}{\hbar \omega}(B-E)\right]}
$$

onde $B=V_{0}\left(R_{0}\right) ; P_{\text {pre }}$ é a probabilidade de o fragmento emitido ser pré-formado no estado fundamental do núcleo que sofre decaimento; o fator $\frac{v}{2 R}$ é o já mencionado número de assaltos, com $v$ sendo a velocidade do fragmento dentro do núcleo $\left(E+V_{0}=\frac{1}{2} \mu v^{2}\right)$ e $R$ é o raio nuclear para $l=0$, onde o subíndice foi omitido por simplicidade. No caso da aplicação real à radioatividade alfa, a aproximação parabólica não é adequada visto que a energia da partícula alfa dentro do núcleo que sofre decaimento é muito menor que a altura da barreira. Para tais energias, é preciso calcular a ação de maneira exata, utilizando uma forma esquemática para a barreira de tunelamento. Uma boa aproximação para o cálculo da ação neste caso é aproximar a barreira de tunelamento para uma barreira coulombiana simples $\left(V_{t}(r)=\frac{Z_{1} Z_{2} e^{2}}{4 \pi \varepsilon_{o} r}\right)$ para distancias maiores que o raio nuclear e considerar um poço quadrado $\left(V_{t}(r)=-V_{0}\right)$ para $r$ menor que o raio nuclear, onde $V_{0}$ é o potencial nuclear que liga a partícula $\alpha$ ao núcleo emissor. Para esta forma da hamiltoniana de tunelamento, a ação de tunelamento $g(l, E)=\sqrt{\frac{8 \mu}{\hbar^{2}}} \int_{r_{1}(l, E)}^{r_{2}(l, E)} d r \sqrt{V_{l}(r)-E}$ pode ser calculada numa forma fechada, utilizando a integral

$$
\int_{R}^{r_{0}} d x \sqrt{\frac{r_{0}}{x}-1}=r_{0}\left[\cos ^{-1} \sqrt{\frac{R}{r_{0}}}-\sqrt{\frac{R}{r_{0}}\left(1-\frac{R}{r_{0}}\right)}\right]
$$


onde $r_{0}$ é o ponto de retorno mais externo, dado por $r_{0}=\frac{Z_{1} Z_{2} e^{2}}{E+V_{0}}$. Visto que $r_{0} \gg R$, fazemos a aproximação $\cos ^{-1}(x) \approx \frac{\pi}{2}-x$, e a integral acima torna-se

$$
\int_{R}^{r_{0}} d x \sqrt{\frac{r_{0}}{x}-1} \approx \frac{\pi}{2} r_{0}-\sqrt{r_{0} R}-R
$$

Com isso, obtemos a conhecida expressão de Gamow para a taxa de tunelamento [25],

$$
R=P_{\text {pre }}\left(\frac{v}{2 R}\right) \exp \left[-2 \pi \eta+\frac{\sqrt{32 Z_{1} Z_{2} \mu R e^{2}}}{\hbar}\right]
$$

onde $\eta$ é o parâmetro de Somerfeld dado por $\eta=\frac{Z_{1} Z_{2} e^{2}}{\hbar v}$, com $v^{2}=\frac{1}{2} \mu\left(E+V_{0}\right)$. É costumeiro associar a energia $E+V_{0}$ à energia liberada com a emissão alfa, a quantidade $Q_{\alpha}$. Isto é simplesmente a diferença entre a massa do núcleo pai e a soma da massa da partícula alfa com a massa do núcleo filho. A famosa equação de Geiger-Nutall é então obtida,

$$
\ln (R)=A-\frac{B}{\sqrt{Q_{\alpha}}}
$$

com $A$ e $B$ identificados com os parâmetros físicos que aparecem na Eq. (2.0.13).

A inclusão do acoplamento com outros graus de liberdade no decaimento por emissão de partículas carregadas se tornou importante com a descoberta da emissão natural de ${ }^{14} \mathrm{C}$ por ${ }^{233} \mathrm{Ra}$ [26,27]. Tal radioatividade seria proibida de acordo com o modelo de penetração em barreira unidimensional de Gamow, o qual se provou tão bem-sucedido no caso da radioatividade alfa. A inclusão de outros graus de liberdade levaria em consideração a hamiltoniana do reservoir e do acoplamento $H_{R}(\zeta)+$ $H_{\text {int }}(r, \zeta)$ em conjunção com o cálculo de tunelamento. Retornaremos a esse ponto mais além.

No contexto da fusão nuclear, um assunto de grande importância no campo da nucleossíntese estelar e na produção de elementos químicos pesados, o cálculo da probabilidade de tunelamento para um dado valor do momento angular aparece na expressão da seção de choque de fusão da seguinte maneira:

$$
\sigma_{F}(E)=\frac{\pi}{k^{2}} \sum_{l=0}^{\infty}(2 l+1) T_{l}(E)
$$


onde $k$ é o valor assintótico do número de onda de movimento relativo $E=\frac{\hbar^{2} k^{2}}{2 \mu}$.

Com a forma de Hill-Wheeler para $T_{l}(E)$, uma expressão analítica pode ser obtida para a seção de choque de fusão $\sigma_{F}(E)$, se $R_{l}$ e $\omega_{l}$ são tomados como independentes do momento angular, i.e. $R_{l} \equiv R_{B}$ e $\omega_{l} \equiv \omega$. Esse é o procedimento seguido por C. Y. Wong [28], que fez a aproximação adicional de substituir a soma em $l$ por uma integral, após introduzir a substituição semi-clássica $l(l+1)=(l+1 / 2)^{2}=\lambda^{2}[29]$. De fato, combinando as Eqs. (2.0.3), (2.0.10) e (2.0.15), obtemos, para a seção de choque de fusão:

$$
\sigma_{F}(E)=\frac{\pi}{k^{2}} \sum_{l=0}^{\infty} \frac{(2 l+1)}{1+\exp \left[\frac{2 \pi}{\hbar \omega}\left(B_{l}-E\right)\right]}
$$

onde $B_{l}=V_{l}\left(R_{B}\right)$, em que $V_{l}$ é definido pela Eq. (2.0.2). Fazendo as aproximações descritas acima, atribuídas a C. Y. Wong, temos

$$
\sigma_{F}(E)=\frac{\pi \hbar^{2}}{\mu E} \int_{0}^{\infty} \frac{d l\left(l+\frac{1}{2}\right)}{1+K_{2} \exp \left[\frac{K_{1} \hbar^{2}\left(l+\frac{1}{2}\right)^{2}}{2 \mu R_{B}^{2}}\right]}
$$

em que $K_{1}=\frac{2 \pi}{\hbar \omega}$ e $K_{2}=\exp \left[\frac{2 \pi}{\hbar \omega}\left(V_{t}\left(R_{B}\right)-E\right)\right]$. Fazendo uma substituição de variáveis, obtemse

$$
\sigma_{F}(E)=R_{B}^{2} \frac{\hbar \omega}{2 E} \int_{K_{2}}^{\exp \left(\frac{-K_{1} \hbar^{2}}{8 \mu R_{B}^{2}}\right)+K_{2}} \frac{d y}{y}
$$

Calculando a integral acima, temos

$$
\sigma_{F}(E)=R_{B}^{2} \frac{\hbar \omega}{2 E} \ln \left\{1+\exp \left[\frac{2 \pi}{\hbar \omega}\left(E-V_{t}\left(R_{B}\right)-\frac{\hbar^{2}\left(0+\frac{1}{2}\right)^{2}}{2 \mu R_{B}^{2}}\right)\right]\right\}
$$

de onde, então, Wong obteve a seguinte expressão simplificada para a seção de choque de fusão:

$$
\sigma_{F}^{W o n g}(E)=R_{B}^{2} \frac{\hbar \omega}{2 E} \ln \left\{1+\exp \left[\frac{2 \pi}{\hbar \omega}(E-B)\right]\right\}
$$

em que $B=V_{0}\left(R_{B}\right)$.

A equação de Wong é apropriada para energias próximas à altura da barreira, devido à limitação da aproximação parabólica para o potencial, na qual ela é baseada. Para energias muito abaixo da 
barreira tal aproximação é falha, visto que normalmente o potencial $V_{l}(r)$ não é simétrico. Não obstante, a fórmula de Wong serve como guia para comparações com resultados mais exatos baseados no método do funcional de influência, ou de canais acoplados.

Correções advindas da reflexão podem ser feitas na aproximação JWKB simples, utilizando o chamado potencial quântico, o qual foi pela primeira vez considerado por Langer [29]. Em uma dimensão, esse potencial aparece quando se utiliza o ansatz JWKB para a função de onda $\psi_{J W K B}(x)=$ $\frac{1}{\sqrt{\frac{d B(x)}{d x}}} \exp ( \pm i B(x))$, que resulta na seguinte forma para a equação de Schrödinger em uma dimensão [29],

$$
\frac{d^{2} \psi_{J W K B}(x)}{d r^{x}}+\left[\left(\frac{d B(x)}{d x}\right)^{2}+\frac{2 m}{\hbar^{2}} V_{q}(x)\right] \psi_{J W K B}(x)=0
$$

onde $\left(\frac{d B(x)}{d x}\right)^{2}=\frac{2 m}{\hbar^{2}}[E-V(x)]$ é o número de onda local, e o potencial quântico $V_{q}(x)$ é dado por

$$
V_{q}(x)=\frac{\hbar^{2}}{2 m}\left[\frac{3\left(\frac{d^{2} B(x)}{d x^{2}}\right)^{2}}{4\left(\frac{d B(x)}{d x}\right)^{2}}-\frac{\frac{d^{3} B(x)}{d x^{3}}}{2 \frac{d B(x)}{d x}}\right]
$$

Por construção, a solução da Eq. (2.0.21) é simplesmente a função de onda JWKB. Para energias acima da altura da barreira, tal função de onda não leva à reflexão e resulta em um fator unitário para a transmissão (tunelamento). É possível obter reflexão a essas energias se for utilizado o potencial quântico em cálculos perturbativos com $\psi_{J W K B}(x)$ usado como uma base "não-perturbada". Quando as derivadas de $B(x)$ são calculadas em termos daquelas do potencial, o potencial quântico torna-se

$$
V_{q}(x)=-\hbar^{2}\left[\frac{5}{32 m}\left(\frac{V^{\prime}(x)}{E-V(x)}\right)^{2}+\frac{V^{\prime \prime}(x)}{8 m[E-V(x)]}\right]
$$

onde $V(x)$ é o potencial de tunelamento unidimensional. Então, pode-se escrever o seguinte potencial efetivo na equação para a função de onda JWKB, $\psi_{J W K B}(x)$, 


$$
V_{J W K B}(x)=V(x)+V_{q}(x)
$$

Por outro lado, a equação de onda para a função de onda exata, $\psi(r)$, que descreve a reflexão quântica é

$$
\frac{d^{2} \psi(x)}{d x^{2}}+\frac{2 m}{\hbar^{2}}[E-V(x)] \psi(x)=0
$$

que pode ser reescrita como

$$
\frac{d^{2} \psi(x)}{d x^{2}}+\frac{2 m}{\hbar^{2}}\left[E-V_{J W K B}(x)\right] \psi(x)=\frac{2 m}{\hbar^{2}} V_{q}(x) \psi(x)
$$

Então, obtém-se a interessante equação de Lippmann-Schwinger:

$$
\left|\psi^{(+)}\right\rangle=\left|\psi_{J W K B}^{(+)}\right\rangle+G_{J W K B}^{(+)} \frac{2 m}{\hbar^{2}} V_{q}\left|\psi^{(+)}\right\rangle
$$

onde $G_{J W K B}^{(+)}\left(x, x^{\prime}\right)$ é a função de Green JWKB unidimensional.

A equação acima pode ser usada para calcular, perturbativamente, a reflexão quântica dentro da aproximação JWKB. O elemento de matriz que descreve exatamente a reflexão quântica (amplitude de reflexão) é dado por

$$
T=\frac{2 m}{\hbar^{2}}\left\langle\psi_{J W K B}^{(-)}\left|V_{q}\right| \psi^{(+)}\right\rangle
$$

O termo frequentemente calculado é o de primeira ordem [30]

$$
T^{(1)}=\left\langle\psi_{J W K B}^{(-)}\left|\frac{2 m}{\hbar^{2}} V_{q}\right| \psi_{J W K B}^{(+)}\right\rangle
$$

onde $+(-)$ representa as soluções JWKB das ondas incidente e transmitida (os sinais da exponencial em $\left.\psi_{J W K B}\right)$. A probabilidade de reflexão é então dada pelo quadrado do módulo da amplitude de reflexão dividido pelo fluxo incidente $\frac{\hbar k}{m}$ : 


$$
R(E)=\left(\frac{2 \mu}{\hbar}\right)^{2}\left|T^{(1)(E)}\right|^{2}=\left(\frac{\mu}{\hbar}\right)^{2}\left|\left\langle\psi_{J W K B}^{(-)}\left|V_{q}\right| \psi_{J W K B}^{(+)}\right\rangle\right|^{2}
$$

Note-se que o potencial quântico acima está imerso em dificuldades; como exemplo disso há o fato de que ele diverge nos pontos de retorno clássicos. Não obstante, ele se faz importante em cálculos de tunelamento. 
CAPÍtulo 3

\section{Tunelamento em Sistemas Complexos e}

\section{Efeito do Meio Externo}

O ponto de discussão inicial do tunelamento em sistemas complexos é o operador hamiltoniano do sistema completo (sub-sistema + reservoir),

$$
H(\mathbf{r}, \zeta)=H_{S}(\mathbf{r})+H_{R}(\zeta)+H_{\text {int }}(r, \zeta)
$$

onde $H_{S}(\mathbf{r})$ é o hamiltoniano do sub-sistema que sofre tunelamento, composto da parte de energia cinética somada ao potencial de tunelamento, $V_{t}(r)$, tomado como sendo esfericamente simétrico:

$$
H_{S}(\mathbf{r})=-\frac{\hbar^{2}}{2 \mu} \nabla^{2}+V_{t}(r)
$$

onde $\mu$ é o parâmetro de massa. Duas abordagens são comumente seguidas no tratamento do hamiltoniano da Eq. (3.0.1). Pode-se introduzir a matriz densidade total do sistema

$$
\rho(\mathbf{r}, \zeta)=|\Psi(\mathbf{r}, \zeta)\rangle \otimes\langle\Psi(\mathbf{r}, \zeta)|
$$

A matriz densidade relevante para o problema de tunelamento é a matriz reduzida 


$$
\hat{\rho}(\mathbf{r})=\operatorname{Tr}_{(\zeta)} \rho(\mathbf{r}, \zeta)
$$

O cálculo da matriz densidade reduzida remete à definição do funcional de influência. Esse procedimento foi seguido por [6-8], assim como por outros no campo da física nuclear [31-33].

O procedimento amplamente utilizado para calcular o tunelamento de acordo com o hamiltoniano da Eq. (3.0.1), baseia-se na expansão da função de onda total do sistema $|\Psi(\mathbf{r}, \zeta)\rangle$ em termos dos autoestados do hamiltoniano do reservoir, $\left|\phi_{n}(\zeta)\right\rangle$. Projeções sobre as funções de onda correspondentes do sub-sistema resultam nas equações de canais acoplados,

$$
\left[E-\epsilon_{n}-H_{S}\right] \psi_{n}(\mathbf{r})=\sum_{m}\left\langle\phi_{n}\left|H_{i n t}\right| \phi_{m}\right\rangle \psi_{m}(\mathbf{r})
$$

onde a equação de Schrödinger para o reservoir $H_{R}\left|\phi_{n}(\zeta)\right\rangle=\epsilon_{n}\left|\phi_{n}(\zeta)\right\rangle$ foi usada.

As equações de canais acoplados acima são numericamente solucionadas utilizando-se condições de contorno apropriadas para as ondas transmitidas em todos os canais não-elásticos e uma soma das ondas transmitidas e incidentes nos canais de entrada. A forma geral da seção de choque de fusão, tomada como sendo a única absorção inerente ao sistema por todos os canais, é dada por $[14,15]$

$$
\sigma_{F}(E)=\sum_{i} \sqrt{\frac{E-\epsilon_{i}}{E}} \sigma_{F}^{i}\left(E-\epsilon_{i}\right)
$$

onde o canal $i$ da seção de choque de fusão é calculado com a função de onda exata naquele canal (solução das equações acopladas acima; consideramos que os canais contém o parâmetro de massa). A expressão acima possivelmente implica um aumento na fusão, ou tunelamento, quando muitos canais são acoplados, ou seja $\sigma_{F}(E) \geq \sigma_{F}^{H_{i n t}=0}(E)$, porém a natureza detalhada desses canais irá definir a validade ou não desta desigualdade (e.g., $\left.\epsilon_{i}<0\right)$.

Em seus papéis seminais, Caldeira e Leggett $[6,7]$ derivaram uma expressão para a probabilidade de tunelamento considerando um meio externo dissipativo, descrito por um coeficiente de fricção $\eta$ e considerando o acoplamento com o reservoir como sendo linear em $q$. A equação clássica de 
movimento para o valor esperado da coordenada de tunelamento $q$ do sub-sistema com parâmetro de massa $M$, sob influência de uma força conservativa é

$$
M \ddot{q}+\eta \dot{q}=-\frac{d V}{d q}
$$

Após escrever a seguinte expressão para a lagrangiana euclidiana,

$$
L_{E}=\frac{1}{2} M \dot{q}^{2}+V(q)+\frac{1}{2} \sum_{\alpha} m_{\alpha}{\dot{x_{\alpha}}}^{2}+\frac{1}{2} \sum_{\alpha} m_{\alpha} \omega_{\alpha}^{2} x_{\alpha}^{2}+q \sum_{\alpha} c_{\alpha} x_{\alpha}
$$

e usando técnicas de integral de trajetória, esses autores obtiveram a expressão abaixo para a probabilidade de tunelamento:

$$
T(\eta)=T(\eta=0) \exp \left[-\frac{A \eta(\delta q)^{2}}{\hbar}\right]
$$

onde $\delta q$ é a largura da barreira, e $A$ é uma constante numérica da ordem da unidade. $\mathrm{O}$ coeficiente de fricção $\eta$ obedece aqui à relação $\sum_{\alpha} \frac{\pi c_{\alpha}^{2}}{2 m_{\alpha} \omega_{\alpha}}=\eta \int d \omega$, onde está subentendido que uma integração sobre as frequências é seguida.

Está claro que o acoplamento com o meio externo diminuirá a probabilidade de tunelamento. Pergunta-se, então, se este efeito seria de natureza geral. Na verdade, um tratamento mais refinado do problema, dado por [31,32], mostrou que além do fator de atenuação exp $\left[-\frac{A \eta(\delta q)^{2}}{\hbar}\right]$, existe uma correção do potencial de tunelamento interior $T(\eta=0)$, que aumenta o tunelamento, especialmente a energias bem abaixo da barreira.

No caso de acoplamento a um meio externo representado por um oscilador harmônico, o tratamento de canais acoplados com frequência zero (aproximação súbita) torna possível o cálculo da probabilidade de transmissão como uma média simples [33],

$$
\left\langle T_{l}(E)\right\rangle_{\zeta} \equiv \int d \zeta\left|\phi_{0}(\zeta)\right|^{2} T_{l}\left[E, V_{l}(r)+H_{\text {int }}(r, \zeta)\right]
$$


onde $T_{l}\left[E, V_{l}(r)+H_{\text {int }}(r, \zeta)\right]$ é a probabilidade de transmissão calculada a energia $E$ com potencial efetivo $V_{l}(r)+H_{\text {int }}(r, \zeta)$, em que $H_{\text {int }}(r, \zeta)$ é o termo do potencial devido ao acoplamento com o reservoir. A função de onda $\phi_{0}(\zeta)$ denota a função de onda no estado fundamental relacionada ao acoplamento com o reservoir. Claramente, funções de onda para estados excitados do acoplamento ao reservoir considerado poderiam ser utilizadas ao invés da função de onda do estado fundamental. A equação acima refere-se ao limite no qual as energias intrínsecas são pequenas quando comparadas à interação de acoplamento, de modo que o hamiltoniano do reservoir é tomado como sendo zero. Neste trabalho consideraremos apenas o caso em que o potencial de acoplamento $H_{\text {int }}(r, \zeta)$ está fixado na posição do máximo da barreira $V_{l}(r)$, i.e. $H_{\text {int }}(r, \zeta)=H_{\text {int }}\left(R_{l}, \zeta\right)$. Embora essa seja uma aproximação muito grosseira, é um primeiro passo no sentido de analisar analiticamente os efeitos da contribuição do potencial de acoplamento no coeficiente de transmissão.

A probabilidade de tunelamento representada pela Eq. (2.0.3), pode, então, ser reescrita para o potencial efetivo $V_{l}(r)+H_{\text {int }}\left(R_{l}, \zeta\right)$ :

$$
T_{l}\left[E, V_{l}(r)+H_{\text {int }}\left(R_{l}, \zeta\right)\right]=\frac{1}{1+\exp \left\{g_{l}\left[E, V_{l}(r)+H_{\text {int }}\left(R_{l}, \zeta\right)\right]\right\}},
$$

com $g_{l}\left[E, V_{l}(r)+H_{\text {int }}\left(R_{l}, \zeta\right)\right]$ dado por:

$$
g_{l}\left[E, V_{l}(r)+H_{\text {int }}\left(R_{l}, \zeta\right)\right]=\sqrt{\frac{8 \mu}{\hbar^{2}}} \int_{r_{1}(l, \zeta)}^{r_{2}(l, \zeta)} d r \sqrt{V_{l}(r)+H_{\text {int }}\left(R_{l}, \zeta\right)-E}
$$

onde $r_{1}(l, \zeta)$ e $r_{2}(l, \zeta)$ são os pontos de retorno clássicos.

A probabilidade de transmissão dada pela Eq. (3.0.11) é simplesmente uma probabilidade de transmissão unidimensional, embora dependa do parâmetro $\zeta$, que representa a coordenada intrínseca devido ao acoplamento com o meio externo.

Para a probabilidade de transmissão acima da barreira, uma discussão detalhada foi dada, por exemplo, em [34], que mostra que $T_{l}\left[E, V_{l}(r)+H_{\text {int }}\left(R_{l}, \zeta\right)\right]$ pode ser escrita exatamente da mesma maneira como para energias abaixo da barreira, se a devida atenção é dada à localização dos pontos de retorno e ao ramo de corte no plano-r imaginário oriundo da raíz quadrada. 
É importante comentar sobre a maneira pela qual os pontos de retorno se movem no plano- $r$ complexo à medida que a energia é aumentada gradualmente, passando da região sob o topo da barreira para a região sobre o topo da barreira. Abaixo da barreira, existem dois pontos de retorno, um exterior, $r_{2}$, e um interior, $r_{1}$. À medida que a energia é aumentada, esses pontos de retorno tornam-se cada vez mais próximos e eventualmente "colidem"quando a energia atinge o valor correspondente ao topo da barreira, passando daí a se distanciarem entre si no plano complexo, tornando-se complexo conjugados um do outro para energias acima da barreira. Isso é mostrado na Figura 4 de [34]. O cálculo da ação de tunelamento, que aqui tem a notação de $g / 2$, é feito ao longo do ramo que garante que tal ação seja real tanto abaixo quanto acima da barreira.

Guiados por Brink e Takigawa [34], Kemble [22], e Miller e Good [23], adaptamos a seguinte prescrição prática para o cálculo numérico da probabilidade de transmissão para energias acima da barreira de potencial:

$$
T_{l}\left[E, V_{l}(r)+H_{i n t}\left(R_{l}, \zeta\right)\right]=\frac{1}{1+\exp \left\{-g_{l}^{a}\left[E, V_{l}(r)+H_{\text {int }}\left(R_{l}, \zeta\right)\right]\right\}},
$$

$\operatorname{com} g_{l}^{a}\left[E, V_{l}(r)+H_{\text {int }}\left(R_{l}, \zeta\right)\right]$ dado por

$$
g_{l}^{a}\left[E, V_{l}(r)+H_{\text {int }}\left(R_{l}, \zeta\right)\right]=\sqrt{\frac{8 \mu}{\hbar^{2}}} \int_{\left|z_{1}(l, \zeta)\right|}^{\left|z_{2}(l, \zeta)\right|} d r \sqrt{V_{l}(r)+H_{\text {int }}\left(R_{l}, \zeta\right)-E}
$$

onde $z_{1}(l, \zeta)$ e $z_{2}(l, \zeta)$ são as raízes complexo-conjugadas $\left(z_{1}=z_{2}^{*}=R_{l}-i \Delta(l, \zeta)\right)$ da equação $V_{l}(r)+H_{\text {int }}\left(R_{l}, \zeta\right)-E=0$, assumindo, como o faz Kemble, uma forma parabólica para $V_{l}(r)$, no processo de determinação dos pontos de retorno.

A Eq. (3.0.10) resulta em um aumento de $T_{l}(E)$, quando comparado ao caso de acoplamento zero com o meio externo. Com a inclusão de frequências finitas não-nulas do oscilador esse aumento é reduzido, visto que tem-se um mecanismo de abaixamento da energia do movimento relativo (dissipação). O recente trabalho intenso neste tópico no contexto da fusão de íons pesados parece indicar um grande aumento da probabilidade de tunelamento a energias próximas à barreira, exceto em casos envolvendo núcleos fracamente ligados, onde o acoplamento ao canal de breakup resulta em certo grau de atenuação da fusão [35]. Adicionalmente, o trabalho recente em fusão de núcleos nor- 
malmente ligados em baixíssimas energias também parece mostrar um apreciável grau de atenuação na taxa de fusão [36,37].

Os resultados acima pedem considerações gerais que evitem modelos específicos, mas que se baseiem em propriedades matemáticas da probabilidade de tunelamento do sub-sistema + meio externo, no limite súbito. Nos voltamos para esta questão na seção que segue. 


\section{Desigualdade de Jensen para}

\section{Probabilidades de Tunelamento}

O propósito deste trabalho é encontrar desigualdades gerais envolvendo probabilidades de tunelamento que comparam o sub-sistema de um sistema com muitos graus de liberdade com relação ao mesmo sistema quando o acoplamento ao meio externo é tomado como uma média. Especifica-

mente, queremos comparar $\left\langle T_{l}\left[E, V_{l}(r)+H_{i n t}\left(R_{l}, \zeta\right)\right]\right\rangle_{\zeta}$ e $T_{l}\left[E, V_{l}(r)+\left\langle H_{i n t}\left(R_{l}, \zeta\right)\right\rangle_{\zeta}\right]$ para o caso geral de reações de fusão.

Para tanto, fazemos uso da desigualdade de Jensen [38], que assegura que para todo funcional $F(f(\zeta))$ de uma função $f$, a seguinte relação é válida

$$
\frac{\int_{a}^{b} d \zeta \phi(\zeta) F(f(\zeta))}{\int_{a}^{b} d \zeta \phi(\zeta)} \geq F\left[\frac{\int_{a}^{b} d \zeta \phi(\zeta) f(\zeta)}{\int_{a}^{b} d \zeta \phi(\zeta)}\right]
$$

se e somente se $F(f)$ é um funcional convexo de $f$ no intervalo [a, b], e $\phi(\zeta)$ é uma função integrável positivamente definida. Se $F(f)$ é um funcional côncavo de $f$, a desigualdade é invertida.

A desigualdade de Jensen é claramente providencial para nosso propósito, no limite súbito (adiabático), onde o hamiltoniano do reservoir é ignorado. A função $\phi$ dentro deste contexto seria a densidade de probabilidade do estado fundamental do reservoir, a qual seria normalizada dentro do 
intervalo físico de interesse. O que resta a ser determinado é a concavidade do funcional, aqui sendo a probabilidade de tunelamento, como função do potencial de acoplamento $H_{\text {int }}(r, \zeta)$.

Trazendo agora a desigualdade de Jensen ao contexto de probabilidade de fusão nuclear, pode-se afirmar que

$$
\left\langle T_{l}(E)\right\rangle_{\zeta} \geq T_{l}\left[E, V_{l}(r)+\left\langle H_{\text {int }}\left(R_{l}, \zeta\right)\right\rangle_{\zeta}\right]
$$

se e somente se $T\left[E, V_{l}(r)+H_{\text {int }}\left(R_{l}, \zeta\right)\right]$ é um funcional convexo de $H_{\text {int }}\left(R_{l}, \zeta\right)$ no intervalo $[a, b]$. Então, faz-se necessário determinar se a probabilidade de transmissão é um funcional côncavo ou convexo de $H_{\text {int }}\left(R_{l}, \zeta\right)$ (onde $H_{\text {int }}\left(R_{l}, \zeta\right)$ é considerado uma variável simples), de modo a fazer uma comparação do tipo da desigualdade (4.0.2). O intervalo $[a, b]$ deve abranger todos os possíveis valores que a coordenada relacionada ao reservoir, $\zeta$, pode assumir.

Vamos introduzir a função $w(\zeta)=E-H_{\text {int }}\left(R_{l}, \zeta\right)$, que será usada em nossos cálculos com a finalidade de tornar a compreensão física mais clara, ou seja, $w(\zeta)$ representará a energia efetiva. Podemos, então, reescrever a Eq. (3.0.11) na forma

$$
T_{l}\left[w(\zeta), V_{l}(r)\right]=\frac{1}{1+\exp \left\{g_{l}\left[w(\zeta), V_{l}(r)\right]\right\}},
$$

onde $w(\zeta)$ representa a "energia efetiva", $V_{l}(r)$ representa o "potencial efetivo"para a onda parcial de número quântico $l$, e a função $g_{l}$ está definida como na Eq. (3.0.12).

Porque $w(\zeta)$ é uma função linear de $H_{\text {int }}\left(R_{l}, \zeta\right)$, o sinal da derivada segunda da probabilidade de tunelamento $T_{l}\left[w(\zeta), V_{l}(r)\right]$, descrita pela equação acima, com relação à função $w(\zeta)$ determinará se $T_{l}$ é um funcional côncavo ou convexo de $H_{i n t}\left(R_{l}, \zeta\right)$ :

$$
\frac{\partial^{2} T_{l}}{\partial w^{2}}=\frac{\exp \left[h_{l}(w)\right]}{\left(1+\exp \left[h_{l}(w)\right]\right)^{3}}\left\{\left(\exp \left[h_{l}(w)\right]-1\right)\left(f_{l}(w)\right)^{2}+\left(\exp \left[h_{l}(w)\right]+1\right)\left(\frac{\partial f_{l}(w)}{\partial w}\right)\right\}
$$

em que $h_{l}(w) \equiv \sqrt{\frac{8 \mu}{\hbar^{2}}} \int_{r_{1}(l, w)}^{r_{2}(l, w)} d r \sqrt{V_{l}(r)-w}$ e $f_{l}(w) \equiv \sqrt{\frac{2 \mu}{\hbar^{2}}} \int_{r_{1}(l, w)}^{r_{2}(l, w)} \frac{d r}{\sqrt{V_{l}(r)-w}}$.

Logo, a derivada segunda descrita pela Eq. (4.0.4) apenas pode ser negativa se a derivada $\left(\frac{\partial f_{l}(w)}{\partial w}\right)$ for negativa. Calculando explicitamente tal derivada, chega-se a uma divergência imprópria, em 
virtude dos limites de integração na função $f_{l}(w)$ serem exatamente as raízes da equação $V_{l}(r)-$ $w=0:$

$$
\begin{gathered}
\frac{\partial}{\partial w}\left\{\int_{r_{1}(l, w)}^{r_{2}(l, w)} \frac{d r}{\sqrt{V_{l}(r)-w}}\right\}=\frac{1}{2} \int_{r_{1}(l, w)}^{r_{2}(l, w)} \frac{d r}{\left(V_{l}(r)-w\right)^{\frac{3}{2}}}+ \\
+\left(\frac{\partial r_{2}(l, w)}{\partial w}\right)\left[\frac{1}{\sqrt{V_{l}(r)-w}}\right]_{r=r_{2}(l, w)}-\left(\frac{\partial r_{1}(l, w)}{\partial w}\right)\left[\frac{1}{\sqrt{V_{l}(r)-w}}\right]_{r=r_{1}(l, w)}
\end{gathered}
$$

No entanto, tal derivada tem um significado físico muito claro, como será discutido mais adiante, e como tal ela não pode ser divergente. Em nosso caso, isso também pode ser verificado numericamente através de gráficos da probabilidade de transmissão, como definida pela Eq. (4.0.3), como função da variável $w$, os quais são todos suaves para os diferentes potenciais que usamos (Figuras $[3-4],[6-16])$. A aparente natureza singular da derivada da função $f_{l}(w)$ é devida à inadequada escolha de variáveis.

Não obstante, podemos tirar vantagem da simetria entre a função $f_{l}(w)$ e a função período definida para movimento finito unidimensional em mecânica clássica:

$$
\tau(E)=\sqrt{2 m} \int_{r_{1}(E)}^{r_{2}(E)} \frac{d r}{\sqrt{E-V(r)}}
$$

onde $m$ é a massa do corpo que descreve o movimento oscilatório e $E$ é sua energia, que é suposta como sendo maior ou igual ao potencial $V(r)$. Os limites da integral aqui também são os pontos de retorno.

A função $f_{l}(w)$ não pode ser sempre analiticamente definida para qualquer função $V(r)$. A vantagem acima mencionada em considerar a simetria entre $f_{l}(w)$ e $\tau(E)$ está, então, na vasta literatura concernente à monotonicidade de funções período em mecânica clássica. 


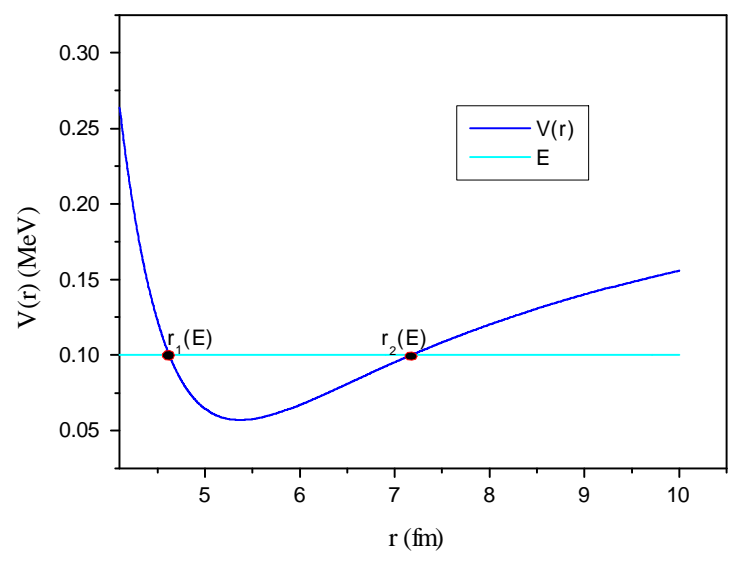

Figura 1: Representação geral de $V(r)$ e E para o movimento clássico oscilatório unidimensional.

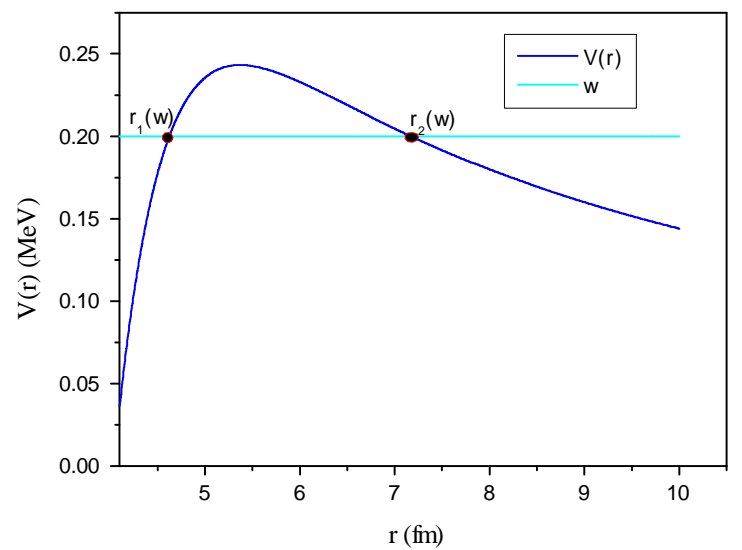

Figura 2: Representação geral de $V(r)$ e de w no caso de tunelamento quântico.

A partir das Figuras (1) e (2), evidenciam-se as semelhanças entre o problema de que tratamos no contexto de tunelamento e o movimento clássico em uma dimensão. Fazendo-se uma rotação e uma translação no gráfico do poço de potencial de um sistema clássico, chegamos ao nosso análogo quântico.

A questão de se a função período é monótona ou não, e no caso de ser monótona, se ela é crescente ou decrescente, não é algo trivial e remonta aos trabalhos de Huygens. Há inúmeros trabalhos que abordam esse problema [39], e que estabelecem alguns critérios para determinar quais potenciais 
resultam em funções período isocrônicas (independentes do valor da energia), ou funções período monotonicamente crescentes ou decrescentes.

\subsection{Desigualdade de Jensen Aplicada à Análise do Efeito de Breakup no Espalhamento Elástico de Núcleos Halo}

Usando o teorema de Peierls anteriormente aludido [20], R. Johnson e C. Goebel derivaram uma desigualdade envolvendo a reflexão acima da barreira de potencial para avaliar o efeito de breakup no espalhamento elástico de núcleos halo [40]. Tal desigualdade clarificou o porquê da seção de choque de reação calculada através do modelo de Glauber ser apreciavelmente menor que aquela calculada usando o limite ótico do modelo, resultando em raios maiores para os núcleos halo, [41]. O resultado de Johnson e Goebel [40] pode ser considerado como uma consequência da desigualdade de Jensen. O caso da fusão de deutérios dentro de um meio metálico pode ser discutido do mesmo modo acima descrito, e encontra-se um aumento na taxa de fusão (fisicamente isto pode ser atribuído à blindagem eletrônica, que resulta em uma diminuição da barreira de potencial).

Em seu trabalho acima citado, Johnson e Goebel consideraram a matriz S exponencial

$$
S_{l}(E, \zeta)=\exp \left[2 i \delta_{l}(E, \zeta)\right]=\exp [f]
$$

onde a diferença de fase $\delta_{l}$, na aproximação JWKB é dada por,

$$
\delta_{l}(E, \zeta)=\lim _{r \rightarrow \infty} \int_{r_{0}}^{r} d r^{\prime}\left(k_{l}\left(r^{\prime}, \zeta\right)-\int_{r_{0}^{(0)}}^{r} d r^{\prime} k_{l}^{(0)}\left(r^{\prime}\right)\right)
$$

Acima, $k_{l}(r, \zeta)$ é o número de onda local dado por

$$
k_{l}(r, \zeta)=\sqrt{\frac{2 \mu}{\hbar^{2}}\left[E-V_{l}(r)-H_{i n t}(r, \zeta)\right]}
$$

$k_{l}^{(0)}(r)$ é o número de onda da partícula livre, $r_{0}$ é o ponto de retorno clássico definido por $k_{l}\left(r_{0}, \zeta\right)=$

0 e $r_{0}^{(0)}$ é o ponto de retorno correspondente ao número de onda livre local. A Eq. (4.1.2) pode, também, ser colocada na forma JWKB, que é mais familiar: 


$$
\delta_{l}(E, \zeta)=\int_{r_{0}}^{\infty} d r k_{l}(r, \zeta)-k r_{0}+\left(l+\frac{1}{2}\right) \pi
$$

Para altas energias, pode-se, na Eq. (4.1.2), expandir o número de onda local em potências de $\frac{V_{l}(r)+H_{\text {int }}(r, \zeta)}{E}$ e reter apenas o termo principal. Isto constitui a aproximação Eikonal considerada por Johnson e Goebel [40]. A essa aproximação corresponde a seguinte diferença de fase,

$$
\delta_{\text {Eikonal }}(E, b, \zeta)=-\frac{\mu}{\hbar^{2} k} \int_{b}^{\infty} r d r \frac{V_{l}(r)+H_{\text {int }}(r, \zeta)}{\sqrt{r^{2}-b^{2}}}
$$

onde o parâmetro de impacto $b=\left(l+\frac{1}{2}\right) / k$. A expressão acima pode ser escrita de forma mais conveniente quando o potencial de acoplamento $H_{i n t}(r, \zeta)$ é escrito na forma separável $F(r) G(\zeta)$ e são usadas coordenadas cilíndricas $(z, b)$, tais que $r=\sqrt{z^{2}+b^{2}}$,

$$
\delta_{\text {Eikonal }}(E, b, \zeta)=-\frac{\mu}{\hbar^{2} k} \int_{-\infty}^{\infty} d z\left[V_{l}(r)+F_{l}(r) G(\zeta)\right]
$$

Consideramos aqui o caso em que o potencial, como também o fator de forma, é puramente absortivo $\left(V(r)=-i W(r)\right.$ e $\left.F_{l}(r)=\frac{d V_{l}(r)}{d r}\right)$. Então, a diferença de fase $\delta_{\text {Eikonal }}$ torna-se um imaginário puro e $f$ real. A partir da desigualdade de Jensen e do fato de que $\delta_{\text {Eikonal }}(E, b, \zeta)$ é uma função linear de $G(\zeta)$, obtemos a desigualdade

$$
\left\langle S_{l}(E, \zeta)\right\rangle_{\zeta} \geqslant \exp \left[-2\left|\left\langle\delta_{\text {Eikonal }}(E, b, \zeta)\right\rangle_{\zeta}\right|\right]
$$

que é o resultado obtido por Johnson e Goebel. Entretanto, a desigualdade acima é válida apenas para diferenças de fase imaginárias. O espalhamento de íons pesados a energias intermediárias, por outro lado, claramente envolve diferenças de fase complexas, e este fato aponta uma limitação inerente ao trabalho de Johnson e Goebel. Tal limitação seria removida se fôssemos a energias muito baixas e considerássemos a fusão dominada por tunelamento quântico (com a integral da ação sendo real, neste caso). 


\subsection{Desigualdade de Jensen Aplicada à Probabilidade de Tunelamento Através de Barreira Retangular}

Para uma barreira de tunelamento retangular, os pontos de retorno não dependem de $w$. O potencial, aqui, é uma constante positiva $V_{0}$ entre os pontos de retorno $r_{1}$ e $r_{2}$, e 0 fora desse intervalo:

$$
\frac{\partial f(w)}{\partial w}=\frac{\partial}{\partial w}\left(\int_{r_{1}}^{r_{2}} \frac{d r}{\sqrt{V_{0}-w}}\right)=\frac{\left(r_{2}-r_{1}\right)}{2\left(V_{0}-w\right)^{\frac{3}{2}}}>0
$$

Então, para tal potencial, a derivada segunda da probabilidade de transmissão com relação a $w$ é positiva para todos os possíveis valores de $w$. Então, usando a desigualdade de Jensen, podemos afirmar o seguinte:

$$
\left\langle T\left[w(\zeta), V_{0}\right]\right\rangle_{\zeta} \geq T\left[\langle w(\zeta)\rangle_{\zeta}, V_{0}\right]
$$

donde

$$
\left\langle T\left[E, V_{0}+H_{\text {int }}\left(R_{l}, \zeta\right)\right]\right\rangle_{\zeta} \geq T\left[E, V_{0}+\left\langle H_{\text {int }}\left(R_{l}, \zeta\right)\right\rangle_{\zeta}\right]
$$

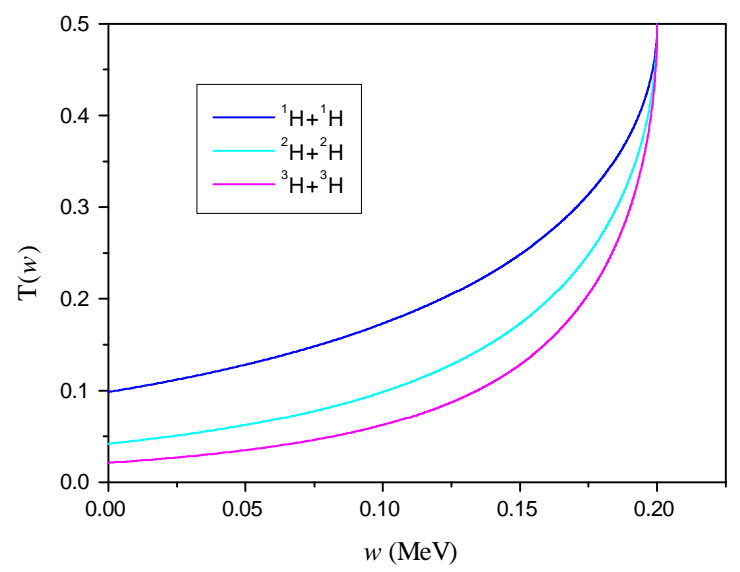

Figura 3: Probabilidade de tunelamento através de uma barreira retangular para os isótopos do hidrogênio. Foi considerada uma barreira retangular de altura $0.2 \mathrm{MeV}$ estendida de 4 a $20 \mathrm{fm}$ a partir do centro do núcleo alvo. Nota-se que todas as curvas são convexas, como esperado. 


\subsection{Desigualdade de Jensen Aplicada à Probabilidade de Tunelamento Através de Barreira de Potencial Isocrônico}

Em mecânica clássica, sistemas isocrônicos são sistemas descritos pelo Hamiltoniano

$$
H=\frac{1}{2} p^{2}+V(q)
$$

tais que admitem uma família de soluções oscilatórias com mesmo período $\tau$, onde $\tau$ é definido pela Eq. (4.0.6). Há inúmeros sistemas isocrônicos na natureza [42]. Aqui, iremos nos limitar aos potenciais isocrônicos racionais. Pode ser provado [43] que, a menos de translações nos eixos $x$ e $y$, todos os potenciais isocrônicos racionais tem a forma:

$$
V(r)=A r^{2}
$$

ou

$$
V(r)=B r^{2}+\frac{C^{2}}{r^{2}}
$$

onde $C$ é uma constante não-nula, $A=\frac{2 \pi^{2}}{\tau^{2}}, B=\frac{\pi^{2}}{2 \tau^{2}}$ e $\tau$ é o período de oscilação. Da primeira classe de potenciais isocrônicos racionais, representada pela Eq. (4.3.1), vê-se que a barreira parabólica, um importante potencial para problemas de tunelamento quântico, é um potencial isocrônico. Diversos trabalhos em tunelamento quântico envolvendo energias próximas à barreira utilizam a aproximação parabólica para o potencial nuclear [44]. Com efeito, para energias altas, o potencial de tunelamento efetivo experimentado pela partícula pode ser aproximado com grande acurácia por uma parábola invertida, como na Eq. (2.0.6),

$$
V_{H W_{l}}(r) \equiv V_{l}\left(R_{l}\right)-\frac{1}{2} \mu \omega_{l}^{2}\left(r-R_{l}\right)^{2}
$$

Pelo termo "isocrônicos", entendemos sistemas que atendem à condição:

$$
\frac{\partial \tau(E)}{\partial E}=\frac{\partial}{\partial E}\left\{\sqrt{2 m} \int_{r_{1}(E)}^{r_{2}(E)} \frac{d r}{\sqrt{E-V(r)}}\right\}=0
$$

de onde, por argumentos de simetria, obtemos

$$
\frac{\partial f(w)}{\partial w}=\frac{\partial}{\partial w}\left\{\sqrt{\frac{2 \mu}{\hbar^{2}}} \int_{r_{1}(w)}^{r_{2}(w)} \frac{d r}{\sqrt{V_{0}-V(r)-w}}\right\}=0
$$


onde $V_{0}$ é uma constante positiva e $V(r)$ é um potencial isocrônico.

Com efeito, usando o potencial parabólico, a função $f_{l}(w)$ torna-se

$$
f_{l}(w)=\sqrt{\frac{2 \mu}{\hbar^{2}}} \int_{r_{1}(l, w)}^{r_{2}(l, w)} \frac{d r}{\sqrt{V_{l}\left(R_{l}\right)-\frac{1}{2} \mu \omega_{l}^{2}\left(r-R_{l}\right)^{2}-w}}
$$

Visto que $r_{1}(l, w)$ e $r_{2}(l, w)$ são raízes da equação $V_{l}\left(R_{l}\right)-\frac{1}{2} \mu \omega_{l}^{2}\left(r-R_{l}\right)^{2}-w=0$, as funções $f_{l}(w)$ e $h_{l}(w)$ podem ser reescritas como

$$
f_{l}(w)=\frac{2}{\hbar \omega_{l}} \int_{r_{1}(l, w)}^{r_{2}(l, w)} \frac{d r}{\sqrt{\left(r-r_{1}(l, w)\right)\left(r_{2}(l, w)-r\right)}}=\frac{2 \pi}{\hbar \omega_{l}}
$$

$\mathrm{e}$

$$
h_{l}(w)=\sqrt{\frac{8 \mu}{\hbar^{2}}} \int_{r_{1}(l, w)}^{r_{2}(l, w)} d r \sqrt{V_{l}\left(R_{l}\right)-\frac{1}{2} \mu \omega_{l}^{2}\left(r-R_{l}\right)^{2}-w}=\frac{2 \pi}{\hbar \omega_{l}}\left[V_{l}\left(R_{l}\right)-w\right]
$$

Da Eq. (4.3.7), temos

$$
\frac{\partial f_{l}(w)}{\partial w}=0
$$

Este resultado é, então, combinado com a Eq. (4.0.4):

$$
\frac{\partial^{2} T_{l}}{\partial w^{2}}=\frac{\exp \left[h_{l}(w)\right]}{\left(1+\exp \left[h_{l}(w)\right]\right)^{3}}\left(\exp \left[h_{l}(w)\right]-1\right)\left(f_{l}(w)\right)^{2}>0
$$

e, portanto,

$$
\left\langle T_{l}\left[w(\zeta), V_{H W_{l}}(r)\right]\right\rangle_{\zeta} \geq T_{l}\left[\langle w(\zeta)\rangle_{\zeta}, V_{H W_{l}}(r)\right]
$$

ou

$$
\begin{aligned}
& \left\langle T_{l}\left[E, V_{l}\left(R_{l}\right)-\frac{1}{2} \mu \omega_{l}^{2}\left(r-R_{l}\right)^{2}+H_{\text {int }}\left(R_{l}, \zeta\right)\right]\right\rangle_{\zeta} \\
& \geq T_{l}\left[E, V_{l}\left(R_{l}\right)-\frac{1}{2} \mu \omega_{l}^{2}\left(r-R_{l}\right)^{2}+\left\langle H_{\text {int }}\left(R_{l}, \zeta\right)\right\rangle_{\zeta}\right]
\end{aligned}
$$

para todas os valores de $l$ e para qualquer valor da massa reduzida do sistema, $\mu$. Isto significa que quando o potencial de tunelamento efetivo tem a forma de uma barreira parabólica, o valor esperado para a probabilidade de tunelamento é maior ou igual à probabilidade de tunelamento 
unidimensional, na qual o potencial de acoplamento $H_{\text {int }}\left(R_{l}, \zeta\right)$ é tomado como uma contribuição média.

À luz deste resultado, podemos discutir como a desigualdade de Jensen clarifica a compreensão do resultado obtido por C. H. Dasso, S. Landowne e A. Winther, em [16]. Neste trabalho, os autores consideram a aproximação em que os $N$ graus de liberdade adicionais de um sistema podem ser degenerados, para fins de diagonalização das equações de Schrödinger acopladas, em apenas um grau de liberdade extra, resultando em duas equações acopladas cuja diagonalização é trivial. Neste processo, o tunelamento através da barreira é sistematizado de forma que a barreira de potencial original, $V^{e f f}(r)$ é bipartida em duas barreiras, a saber $V^{e f f}(r) \pm \sqrt{N} V^{c p l}(r)$, ou seja, uma das barreiras oriundas do processo de acoplamento do grau de liberdade extra tem seu topo mais alto que a original, enquanto a outra é mais baixa que a original. Considerando o potencial de acoplamento $V^{c p l}$ como sendo independente de $r$, que é a aproximação que fazemos neste trabalho, temos que as duas barreiras resultantes do processo de bipartição serão idênticas à original em forma, apenas deslocadas para cima e para baixo no eixo- $y$. Ora, isso é análogo a considerar que a barreira original permanece a mesma, enquanto que a energia com a qual o processo de tunelamento ocorrerá é equipartida em duas, uma maior do que a energia original por uma quantidade $\sqrt{N} V^{c p l}$, e outra menor que a original pela mesma quantidade. Os autores então mostram que a probabilidade de transmissão total, dada pela média aritmética das transmissões calculadas nos sistemas oriundos da bipartição da barreira de potencial, é maior ou igual à probabilidade de tunelamento original :

$$
T_{t o t} \equiv \frac{1}{2}\left[T_{+}+T_{-}\right] \geqslant T
$$

onde a probabilidade de tunelamento $T$ é calculada com a barreira de potencial original, $V^{e f f}(r)$, e $T_{+}, T_{-}$são calculadas com a s barreiras de potencial $V^{e f f}(r)+\sqrt{N} V^{c p l}$ e $V^{e f f}(r)-\sqrt{N} V^{c p l}$, respectivamente. Com efeito, considerando a probabilidade de tunelamento como uma função convexa da "energia efetiva" $E=E_{0} \pm \sqrt{N} V^{c p l}\left(E_{0}\right.$ sendo a energia original na qual o processo de tunelamento ocorre), como demonstramos no início desta seção ser o caso para barreiras de potencial aproximadamente parabólicas, então pode-se afirmar, através da desigualdade de Jensen, que 
$\langle T(E)\rangle \geqslant T(\langle E\rangle)$. Ora, se a média considerada é a média aritmética (discreta) e tomamos apenas dois pontos na curva de $T(E)$, chegamos trivialmente ao resultado representado pela Eq. (4.3.12).

Calculando a derivada segunda da probabilidade de transmissão representada pelas Eqs. (3.0.13) e (3.0.14) e seguindo o mesmo procedimento descrito acima, encontra-se que

$$
\left\langle T_{l}\left[E, V_{H W l}(r)+H_{\text {int }}\left(R_{l}, \zeta\right)\right]\right\rangle_{\zeta} \leqslant T_{l}\left[E, V_{H W l}(r)+\left\langle H_{\text {int }}\left(R_{l}, \zeta\right)\right\rangle_{\zeta}\right]
$$

para energias acima da barreira de potencial. Disto infere-se que para a aproximação parabólica para o potencial, todos os sistemas mostram transmissão reduzida para energias acima da barreira.

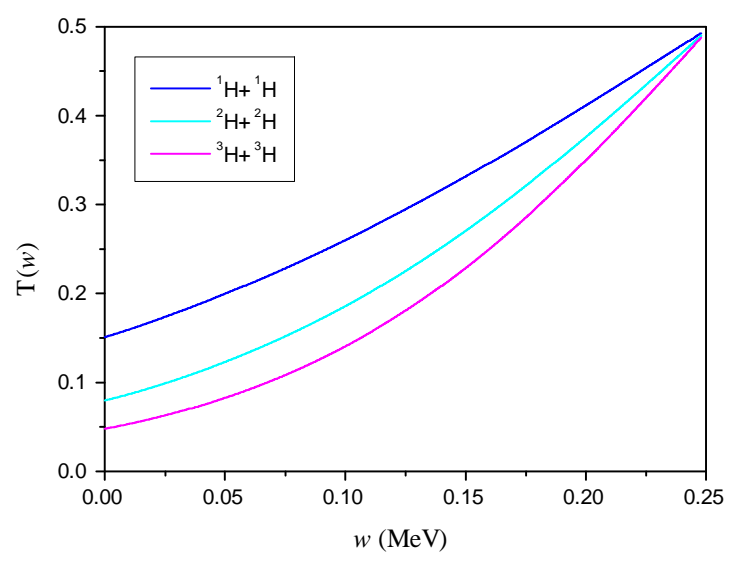

Figura 4: Probabilidade de tunelamento através de uma barreira parabólica para os isótopos do hidrogênio. A barreira considerada, cuja altura era $0.25 \mathrm{MeV}$, foi $V(r)=-0.005 r^{2}+0.071 r$ para $0 \leqslant r \leqslant 14$ fm e 0 fora desse intervalo. Todas as curvas são convexas, como esperado analiticamente.

A segunda classe de potenciais isocrônicos representada em sua forma mais geral pela Eq. (4.3.2) também é muito interessante do ponto de vista de tunelamento quântico devido ao caráter assimétrico dessa curva de potencial, tendo em vista que a maioria das barreiras reais de tunelamento são assimétricas. Fazendo uma reflexão com relação ao eixo- $x$ no gráfico de um potencial da forma da Eq. (4.3.2), seguida de translações nos eixos $x$ e $y$, teremos a barreira de potencial de tunelamento quântico análoga ao poço de potencial do problema clássico correspondente. Um exemplo de tal barreira de potencial pode ser visto na figura abaixo: 


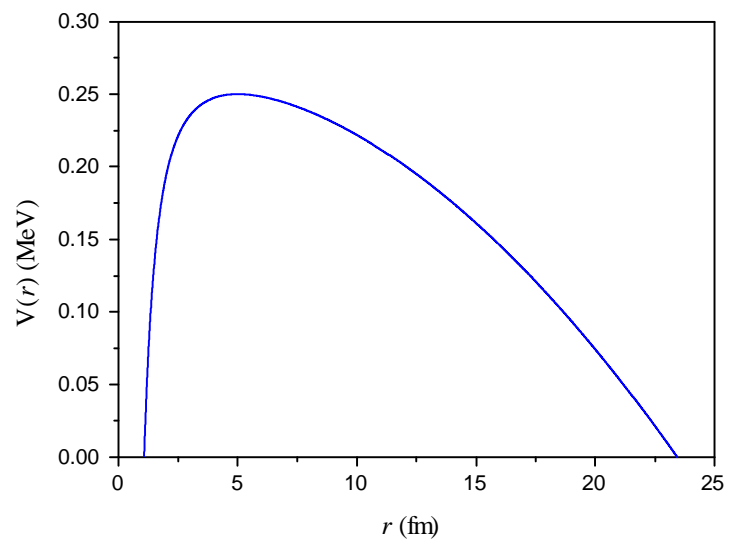

Figura 5: Exemplo de potencial isocrônico da forma $V(r)=V_{0}-B r^{2}-\frac{C^{2}}{r^{2}}$. Neste exemplo,

$$
V_{0}=0.275, B=0.0005 \text { e } C^{2}=0.3125 \text {. }
$$

Procedendo de maneira análoga ao caso da barreira parabólica, podemos afirmar que

$$
\left\langle T\left[E, V_{0}-B r^{2}-\frac{C^{2}}{r^{2}}+H_{\text {int }}\left(R_{l}, \zeta\right)\right]\right\rangle_{\zeta} \geq T\left[E, V_{0}-B r^{2}-\frac{C^{2}}{r^{2}}+\left\langle H_{\text {int }}\left(R_{l}, \zeta\right)\right\rangle_{\zeta}\right]
$$

A inequação (4.3.14) implica que as curvas da probabilidade de transmissão com relação à função $w=E-H_{\text {int }}(R, \zeta)$ devem ser convexas independentemente da massa reduzida do sistema, ou seja, independentemente do par de átomos que sofrerá fusão atrvés de tal potencial. Isso pode ser confirmado na figura que se segue:

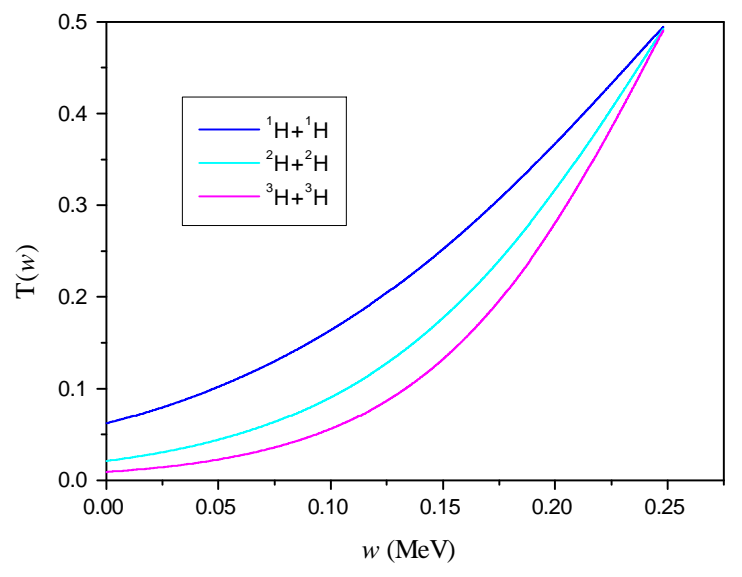


Figura 6: Probabilidade de tunelamento através da barreira mostrada na Figura 5 para os isótopos do hidrogênio.

\subsection{Desigualdade de Jensen Aplicada à Probabilidade de Tunelamento Através de Barreira de Potencial Coulombiano}

Em problemas de fusão nuclear, especialmente em baixas energias, a barreira de tunelamento muito frequentemente é aproximada pela barreira coulombiana acompanhada de um poço retangular abrupto que representa a região efetiva de matéria nuclear. Tal potencial pode ser representado analiticamente da seguinte maneira:

$$
V(r)=-V_{0} \theta\left(r_{0}-r\right)+\frac{C_{1}}{r} \theta\left(r-r_{0}\right)
$$

onde $V_{0}$ é uma constante positiva, $r_{0}$ é o raio nuclear efetivo e $C_{1}=\frac{Z_{1} Z_{2} e^{2}}{4 \pi \epsilon_{0}}$, em que $e$ é a carga elétrica fundamental. Para tal potencial, temos que $r_{1}(w)=r_{0}$ :

$$
f(w) \equiv \sqrt{\frac{2 \mu}{\hbar^{2}}} \int_{r_{0}}^{r_{2}(w)} \frac{d r}{\sqrt{\frac{C_{1}}{r}-w}}
$$

Então, computando a derivada da função $f(w)$ com relação a $w$, temos:

$$
\frac{\partial f(w)}{\partial w}=-\frac{3 C_{1}}{w^{\frac{5}{2}}} \operatorname{ArcTan}\left\{\sqrt{\frac{\sqrt{\frac{C_{1}}{w r_{0}}}-1}{\sqrt{\frac{C_{1}}{w r_{0}}}+1}}\right\}-\frac{r_{0}}{2 w^{\frac{3}{2}}} \frac{\left(\frac{4 C_{1}}{w r_{0}}-1\right)}{\sqrt{\frac{C_{1}}{w r_{0}}-1}}
$$

Visto que $0<w<\frac{C_{1}}{r_{0}}$, temos que $\frac{C_{1}}{w r_{0}}>1$, de modo que o lado direito da Eq. (4.4.1) resulta em um valor negativo para qualquer valor permitido de $w$ e qualquer valor admitido de $r_{0}\left(r_{0}>0\right)$. Usando esse resultado de maneira qualitativa (apenas usando o fato de que, para o potencial coulombiano, $\left.\frac{\partial f(w)}{\partial w}<0\right)$ na Eq. (4.0.4), vemos que o sinal da derivada segunda da probabilidade de transmissão com relação a $w$ ainda não fica determinado. Calculando numericamente a probabilidade de transmissão $T(w)$ com relação a $w$, onde $T(w)$ é dado pela Eq. (2.0.3), obtivemos os gráficos mostrados a seguir: 


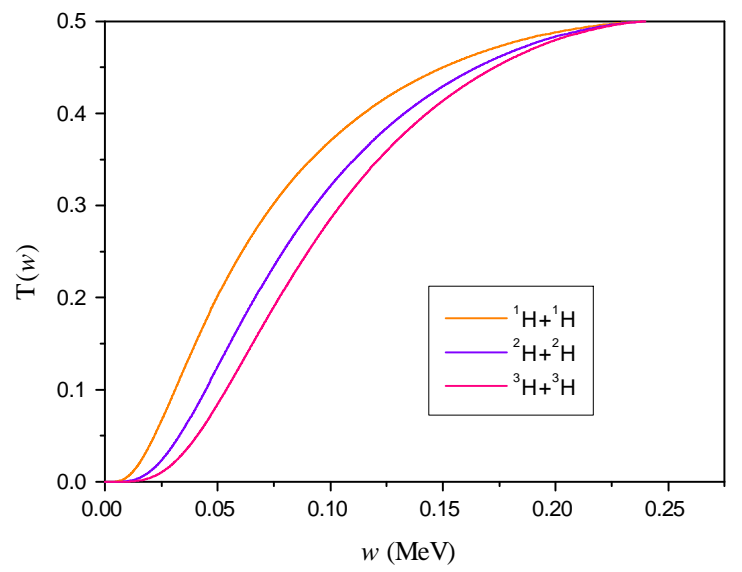

Figura 7: Probabilidade de tunelamento através da barreira coulombiana para os isótopos do hidrogênio. É notada a mudança de concavidade nas curvas à medida que w cresce. Aqui utilizamos $r_{0}=6 \mathrm{fme} \frac{e^{2}}{4 \pi \varepsilon_{0}}=1.44 \mathrm{MeV} \cdot \mathrm{fm}$.

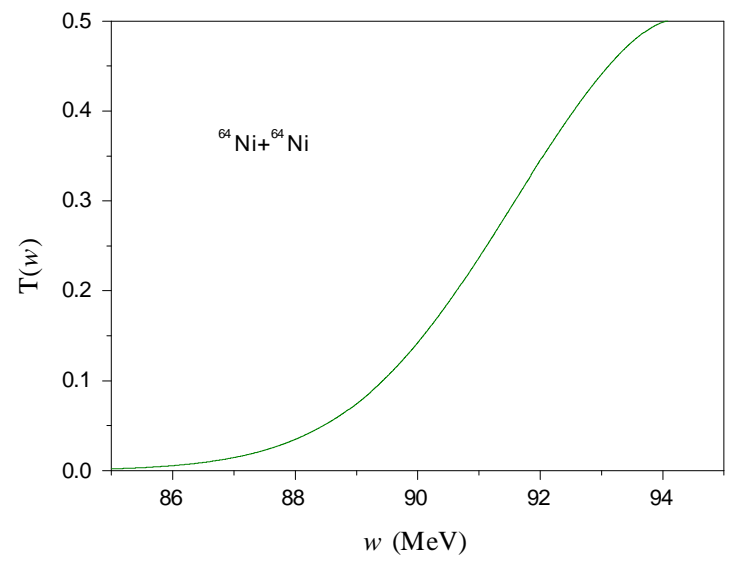

Figura 8: Probabilidade de tunelamento através da barreira coulombiana para dois núcleos de ${ }^{64} \mathrm{Ni}$. Note-se que a região onde a curva é convexa é proporcionalmente maior em comparação com a Figura7. Aqui utilizamos $r_{0}=12 \mathrm{fme} \frac{e^{2}}{4 \pi \varepsilon_{0}}=1.44 \mathrm{MeV} \cdot \mathrm{fm}$. 


\subsection{Desigualdade de Jensen Aplicada à Probabilidade de Tunelamento Através de Barreira de Potenciais Nuclear, Coulombiano e Cen- trífugo Combinados}

\subsubsection{Desigualdades Para Fusão em Baixas Energias}

Abordaremos agora a aplicação da desigualdade de Jensen para o caso de baixas energias. Por "baixas energias"entendemos pequenos valores da função $w(\zeta)=E-F\left(R_{l}\right) G(\zeta)$. A aproximação parabólica para a barreira de potencial não é adequada para este caso, no qual usaremos uma interação efetiva íon-íon da forma:

$$
V_{l}(r) \equiv V_{N}(r)+\frac{Z_{1} Z_{2} e^{2}}{4 \pi \varepsilon_{0} r}+\frac{\hbar^{2} l(l+1)}{2 \mu r^{2}}
$$

em que $V_{N}(r)$ é o potencial nuclear.

Agora, para valores pequenos de $w(\zeta)$, fazemos a aproximação:

$$
\begin{gathered}
\left(\exp \left[\sqrt{\frac{8 \mu}{\hbar^{2}}} \int_{r_{1}(l, w)}^{r_{2}(l, w)} d r \sqrt{V_{l}(r)-w}\right]-1\right) \approx\left(\exp \left[\sqrt{\frac{8 \mu}{\hbar^{2}}} \int_{r_{1}(l, w)}^{r_{2}(l, w)} d r \sqrt{V_{l}(r)-w}\right]+1\right) \\
\approx \exp \left[\sqrt{\frac{8 \mu}{\hbar^{2}}} \int_{r_{1}(l, w)}^{r_{2}(l, w)} d r \sqrt{V_{l}(r)-w}\right]
\end{gathered}
$$

de modo que, para tais valores de $w(\zeta)$, é possível aproximar a Eq. (4.0.4) da seguinte maneira:

$$
\frac{\partial^{2} T_{l}}{\partial w^{2}} \approx \frac{\exp \left[2 h_{l}(w)\right]}{\left(1+\exp \left[h_{l}(w)\right]\right)^{3}}\left\{\left(f_{l}(w)\right)^{2}+\left(\frac{\partial f_{l}(w)}{\partial w}\right)\right\}
$$

em que $f_{l}(w)$ e $h_{l}(w)$ estão definidos na Eq. (4.0.4). A partir da equação acima, vemos que o sinal de $\frac{\partial^{2} T_{l}}{\partial w^{2}}$ dependerá exclusivamente do sinal do termo $\left\{\left(f_{l}(w)\right)^{2}+\left(\frac{\partial f_{l}(w)}{\partial w}\right)\right\}$. Mostraremos que tal termo, considerando a barreira de potencial para reação de fusão com a qual estamos lidando (Eq. (4.5.1)), é sempre positivo quando $w(\zeta)$ tende a zero. Para fazer isso, assumimos primeiramente a seguinte hipótese nula: $\left\{\left(f_{l}(w)\right)^{2}+\left(\frac{\partial f_{l}(w)}{\partial w}\right)\right\}_{w \rightarrow 0} \leqslant 0$. Então,

$$
\lim _{w \rightarrow 0}\left\{-\frac{d}{d w}\left(\frac{1}{f(w)}\right)\right\} \leqslant-1 \Rightarrow 1 \geqslant \lim _{w \rightarrow 0}\{w f(w)\} \Rightarrow 1 \geqslant \lim _{w \rightarrow 0}\left\{\sqrt{\frac{2 \mu}{\hbar^{2}}} w \int_{r_{1}(l, w)}^{r_{2}(l, w)} \frac{d r}{\sqrt{V_{l}(r)-w}}\right\}
$$


Agora, fazemos

$$
\int_{r_{1}(l, w)}^{r_{2}(l, w)} \frac{d r}{\sqrt{V_{l}(r)-w}}=\int_{r_{1}(l, w)}^{r^{*}} \frac{d r}{\sqrt{V_{l}(r)-w}}+\int_{r^{*}}^{r_{2}(l, w)} \frac{d r}{\sqrt{V_{l}(r)-w}}
$$

em que $r_{1}(l, w)<r^{*}<r_{2}(l, w)$. Aqui, $r^{*}$ é escolhido como sendo suficientemente maior do que o raio nuclear efetivo para reação de fusão, de modo que a uma tal distância, o potencial nuclear atrativo, $V_{N}(r)$, seja desprezível. Então,

$$
1 \geqslant \lim _{w \rightarrow 0}\left\{\sqrt{\frac{2 \mu}{\hbar^{2}}} w I_{1}\right\}+\lim _{w \rightarrow 0}\left\{\sqrt{\frac{2 \mu}{\hbar^{2}}} w I_{2}\right\}
$$

onde $I_{1} \equiv \int_{r_{1}(l, w)}^{r^{*}} \frac{d r}{\sqrt{V_{l}(r)-w}}$ e $I_{2} \equiv \int_{r^{*}}^{r_{2}(l, w)} \frac{d r}{\sqrt{V_{l}(r)-w}}$. Claramente a integral $I_{1}$ é limitada para $w \rightarrow 0$, e, portanto, $\lim _{w \rightarrow 0}\left\{\sqrt{\frac{2 \mu}{\hbar^{2}}} w I_{1}\right\}=0$. Isto nos deixa com a desigualdade:

$$
1 \geqslant \lim _{w \rightarrow 0}\left\{\sqrt{\frac{2 \mu}{\hbar^{2}}} w I_{2}\right\}
$$

Fazendo uma mudança de variáveis, a saber, $y=V_{l}(r)-w$, obtemos, para $I_{2}$ :

$$
I_{2}=\int_{V\left(r^{*}\right)-w}^{0} \frac{d y}{\sqrt{y}} \frac{d V_{l}^{-1}(y+w)}{d y}
$$

Visto que $r^{*}$ é tomado como sendo muito maior que o raio nuclear efetivo, a contribuição para o potencial total $V_{l}(r)$ do potencial atrativo de Woods-Saxon pode ser desprezada no intervalo $\left(r^{*}, r_{2}(l, w)\right)$. Tendo isto em conta, no cálculo de $I_{2}$ fazemos a seguinte aproximação:

$$
V_{l}(r) \approx \frac{C_{1}}{r}+\frac{C_{2 l}}{r^{2}}
$$

em que $C_{1}=\frac{Z_{1} Z_{2} e^{2}}{4 \pi \epsilon_{0}}$ e $C_{2 l}=\frac{\hbar^{2} l(l+1)}{2 \mu}$. Claramente, $C_{1}$ e $C_{2 l}$ são ambas não-negativas. Assumiremos primeiro que $l \neq 0$, ou seja, que $C_{2 l}$ é uma constante estritamente positiva. Da Eq. (4.5.5), temos

$$
r=\frac{C_{1}+\sqrt{C_{1}^{2}+4(y+w) C_{2 l}}}{2(y+w)}
$$


donde

$$
I_{2}=\int_{\frac{C_{1}}{r^{*}}+\frac{C_{2 l}}{r^{* 2}}-w}^{0} d y\left[\frac{C_{2 l}}{(y+w) \sqrt{y\left(C_{1}^{2}+4(y+w) C_{2 l}\right)}}-\frac{C_{1}+\sqrt{C_{1}^{2}+4(y+w) C_{2 l}}}{2 \sqrt{y}(y+w)^{2}}\right]
$$

Como nosso objetivo aqui é a verificação da desigualdade (4.5.4), não nos ateremos aqui ao cálculo exato da integral representada pela Eq. (4.5.6). Ao invés disso, usaremos um atalho matemático fundamentado na seguinte desigualdade, que obtem-se derivando parcialmente a integral $I_{2}$ com relação à constante $C_{1}$, onde $C_{1}$ aqui é tomada como uma variável:

$$
\frac{\partial I_{2}}{\partial C_{1}}>0
$$

Uma consequência direta desse fato é que $\lim _{C_{1} \rightarrow 0}\left\{I_{2}\left(C_{1}\right)\right\} \leqslant I_{2}\left(C_{1}\right)$, já que $C_{1}$ é positiva. Logo,

$$
\begin{gathered}
\lim _{C_{1} \rightarrow 0}\left\{I_{2}\left(C_{1}\right)\right\}=\frac{\sqrt{C_{2 l}}}{2} \int_{0}^{\frac{C_{2 l}}{r^{* 2}-w}} \frac{d y}{\sqrt{y}(y+w)^{\frac{3}{2}}} \leqslant I_{2} \\
\Rightarrow \frac{\sqrt{C_{2 l}}}{w} \sqrt{1-\frac{w\left(r^{*}\right)^{2}}{C_{2 l}}} \leqslant I_{2}
\end{gathered}
$$

Multiplicando ambos os lados da inequação acima por $w$ e tomando o limite $w \rightarrow 0$, obtemos

$$
\sqrt{C_{2 l}} \leqslant \lim _{w \rightarrow 0}\left\{w I_{2}(w)\right\}
$$

Combinando o último resultado com a inequação (4.5.4),

$$
1 \geqslant \sqrt{\frac{2 \mu}{\hbar^{2}}} \lim _{w \rightarrow 0}\left\{w I_{2}(w)\right\} \geqslant \sqrt{\frac{2 \mu}{\hbar^{2}} C_{2 l}}
$$

o que nos leva ao resultado absurdo de que $\sqrt{l(l+1)} \leqslant 1$, já que $C_{2 l}=\frac{\hbar^{2} l(l+1)}{2 \mu}$. Por hipótese, $l \neq 0$, de modo que o valor mínimo para o termo $\sqrt{l(l+1)}$ é $\sqrt{2}$. Isto nos leva a uma contradição, significando que nossa hipótese nula inicial, $\left\{\left(f_{l}(w)\right)^{2}+\left(\frac{\partial f_{l}(w)}{\partial w}\right)\right\}_{w \rightarrow 0} \leqslant 0$ é falsa.

Examinemos agora o caso $l=0$, caso em que o potencial $V_{l}(r)$ usado em $I_{2}$ será apenas o potencial de Coulomb:

$$
V_{0}(r) \approx \frac{C_{1}}{r}
$$


Com o potencial acima, a integral $I_{2}$ torna-se, para $w \rightarrow 0$ :

$$
I_{2}=\frac{r^{*}}{\sqrt{w}} \sqrt{\frac{C_{1}}{w r^{*}}-1}+\frac{2 C_{1}}{w^{\frac{3}{2}}} \arctan \left\{\exp \left[\operatorname{arccosh}\left(\sqrt{\frac{C_{1}}{w r^{*}}}\right)\right]\right\}-\frac{\pi C_{1}}{2 w^{\frac{3}{2}}}
$$

Multiplicando ambos os lados da Eq. (4.5.8) por $w$ e tomando o limite $w \rightarrow 0$, encontramos que $\lim _{w \rightarrow 0}\left\{w I_{2}(w)\right\}=\infty$, de forma que novamente a desigualdade (4.5.4) não pode ser satisfeita para o caso da onda parcial $\operatorname{com} l=0$, ratificando o fato de que a hipótese nula $\left\{\left(f_{l}(w)\right)^{2}+\left(\frac{\partial f_{l}(w)}{\partial w}\right)\right\}_{w \rightarrow 0} \leqslant$ 0 é falsa. Retomando a Eq. (4.5.2), temos

$$
\lim _{w \rightarrow 0}\left\{\frac{\partial^{2} T_{l}}{\partial w^{2}}\right\}=\lim _{w \rightarrow 0}\left\{\frac{\exp \left[2 h_{l}(w)\right]}{\left(1+\exp \left[h_{l}(w)\right]\right)^{3}}\left\{\left(f_{l}(w)\right)^{2}+\left(\frac{\partial f_{l}(w)}{\partial w}\right)\right\}\right\}>0
$$

o que implica na seguinte desigualdade de Jensen:

$$
\left\langle T_{l}\left[E, V_{l}(r)+H_{\text {int }}\left(R_{l}, \zeta\right)\right]\right\rangle_{\zeta} \leqslant T_{l}\left[E, V_{l}(r)+\left\langle H_{\text {int }}\left(R_{l}, \zeta\right)\right\rangle_{\zeta}\right]
$$

para $\left\{E-H_{\text {int }}\left(R_{l}, \zeta\right)\right\} \rightarrow 0$ e onde $V_{l}(r)$ é dado pela Eq. (4.5.1).

Este resultado bastante geral implica que qualquer que seja a forma analítica do potencial nuclear adotado, o gráfico da probabilidade de transmissão versus $w(\zeta)$, onde $w(\zeta)=E-F\left(R_{l}\right) G(\zeta)$, é convexo para valores suficientemente pequenos de $w$, acarretando num aumento no tunelamento para tais valores.

Até aqui, nos concentramos no coeficiente de transmissão para a onda parcial de número quântico l. Os dados experimentais para fusão ocorrendo via processo de tunelamento quântico, por outro lado, são representados pela seção de choque de fusão definida por:

$$
\sigma_{F}(E)=\frac{\pi \hbar^{2}}{2 \mu E} \sum_{l=0}^{\infty}(2 l+1) T_{l}(E)=\sum_{l=0}^{\infty} \sigma_{l}(E)
$$

A partir da Eq. (4.5.10), vemos que a dependência de $\sigma_{F}(E)$ com relação ao acoplamento $H_{\text {int }}\left(R_{l}, \zeta\right)$ está apenas nos termos $T_{l}(E)$. Então, se for possível afirmar que, por exemplo, $T_{l}(E)$ é um funcional convexo de $H_{\text {int }}\left(R_{l}, \zeta\right)$ para todos os valores do número quântico $l$, então também se poderá afirmar que $\sigma_{F}(E)$ é um funcional convexo de $H_{\text {int }}\left(R_{l}, \zeta\right)$. Isto dá suporte à idéia geral que há um 
aumento na seção de choque de fusão quando o acoplamento aos graus de liberdade do reservoir são levados em consideração, ou seja,

$$
\left\langle\sigma_{F}(w(\zeta))\right\rangle_{\zeta} \geq \sigma_{F}\left(\langle w(\zeta)\rangle_{\zeta}\right)
$$

para o processo de fusão ocorrendo via tunelamento através de uma barreira de potencial.

Com efeito, para energias muito abaixo do topo da barreira, o coeficiente de transmissão, ou probabilidade de tunelamento, pode ser aproximado por uma exponencial, visto que a ação na aproximação uniforme para o coeficiente de transmissão é pequena:

$$
\sigma_{F}(E)=\frac{\pi \hbar^{2}}{2 \mu E} T_{0}(E)=\frac{\pi \hbar^{2}}{2 \mu E} \exp \left[-g_{0}\left(E, V(r)+H_{i n t}\left(R_{l}, \zeta\right)\right)\right]
$$

De maneira análoga ao que foi demonstrado no início desta subseção, mostra-se que $\sigma_{F}(E)$, como definida na Eq. (4.5.12) é convexa em $H_{\text {int }}\left(R_{l}, \zeta\right)$ para $w(\zeta) \rightarrow 0$, e logo sua média sobre $\zeta$ é maior ou igual que $\sigma_{F}(E)$ calculada com $\left\langle H_{\text {int }}\left(R_{l}, \zeta\right)\right\rangle_{\zeta}$. Portanto, podemos afirmar que

$$
\left\langle\exp \left[-g_{0}\left(E, V(r)+H_{\text {int }}\left(R_{l}, \zeta\right)\right)\right]\right\rangle_{\zeta} \geq \exp \left[-g_{0}\left(E, V(r)+\left\langle H_{\text {int }}\left(R_{l}, \zeta\right)\right\rangle_{\zeta}\right)\right]
$$

que representa a versão de tunelamento, num limite de baixas, da desigualdade de Johnson e Goebel concernente ao elemento da matriz-S eikonal do espalhamento elástico de núcleos halo.

Atualmente, é bem sabido que um grande aumento em $\sigma_{F}$, com relação ao limite de não-acoplamento, tem sido observado para a maioria dos sistemas de fusão de íons pesados a energias próximas do limite da barreira [45]. Recentemente, foi reportado que a energias muito baixas, este aumento é reduzido [36] (infelizmente, este efeito tem sido amplamente chamado de "diminuição do tunelamento", que não deve ser confundido com o que chamamos de diminuição da probabilidade de tunelamento neste trabalho, que seria um comportamento côncavo $T_{l}(w(\zeta))$ como função de $\left.H_{\text {int }}\left(R_{l}, \zeta\right)\right)$. 


\subsubsection{Desigualdades Para Todo o Espectro de Energia}

De acordo com a discussão da seção anterior, esperamos que, no contexto das aproximações acerca das quais ponderamos neste trabalho, todos os gráficos da probabilidade de tunelamento em função de $w$ mostrem uma curva convexa ao menos para pequenos valores de $w$. Por outro lado, remontando à discussão da seção (3.3), toda vez que a barreira de tunelamento puder bem ser aproximada por uma parábola, então também um comportamento convexo da curva da probabilidade de transmissão é esperado para energias abaixo do topo da barreira de potencial, enquanto que um comportamento côncavo de tal curva é esperado para energias acima do topo da barreira de potencial.

Visando à verificação da acurácia dos resultados analíticos que obtivemos até agora, plotamos a probabilidade de fusão, $T_{l}$, como definida pelas Eqs. (3.0.11), (3.0.12) e pelas Eqs. (3.0.13), (3.0.14); respectivamente para valores de $w$ abaixo e acima do topo da barreira. O modo como foram feitos os cálculos envolvendo os pontos de retorno complexos presentes na Eq. (3.0.14) está descrito em detalhe no Apêndice. A barreira de potencial aqui utilizada foi a interação efetiva íon-íon representada abaixo, onde a interação nuclear foi tida como do tipo Woods-Saxon:

$$
V_{l}(r)=\frac{-V_{0}}{1+\exp \left(\frac{r-R_{0}}{\alpha_{p}}\right)}+\frac{Z_{1} Z_{2} e^{2}}{4 \pi \varepsilon_{0} r}+\frac{\hbar^{2} l(l+1)}{2 \mu r^{2}}
$$

onde a difusividade $a_{p}$ foi tomada como sendo $0.65 \mathrm{fm}$, o raio nuclear efetivo do sistema foi calculado como $R_{0}=1.31\left(\sqrt[3]{A_{1}}+\sqrt[3]{A_{2}}\right)-1.68 \mathrm{fm}$, onde $A_{1}$ e $A_{2}$ são os números de massa dos núcleos envolvidos na reação de fusão, e o parâmetro $V_{0}$ foi escolhido de modo que o potencial Woods-Saxon e o potencial de São Paulo coincidissem na coordenada correspondente à superfície nuclear efetiva $[46,47]$.

Nas Figuras (9) e (10) temos a probabilidade de tunelamento para os sistemas ${ }^{64} \mathrm{Ni}+{ }^{64} \mathrm{Ni} \mathrm{e}{ }^{16} \mathrm{O}+{ }^{150} \mathrm{Sm}$, respectivamente. Ambos os gráficos foram computados para a onda parcial com $l=0$. 


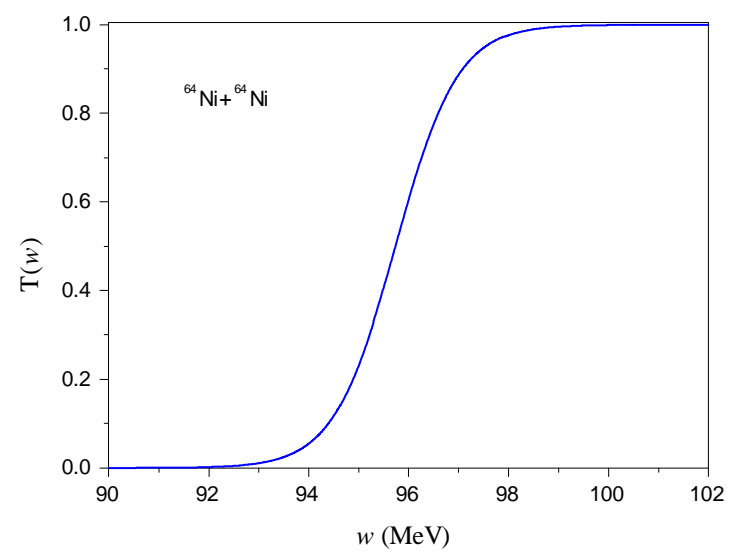

Figura 9: Probabilidade de transmissão versus a função $w(\zeta)$ para o sistema ${ }^{64} \mathrm{Ni}+{ }^{64} \mathrm{Ni}$, para $l=0$. Na região classicamente proibida, i.e. quando $0 \leqslant T(w) \leqslant 0.5$, a curva mostra uma dependência convexa do funcional probabilidade de tunelamento com relação à função $w(\zeta)$, enquanto que na região classicamente permitida, a curva torna-se côncava. Desde que a concavidade muda, a desigualdade de Jensen é revertida quando se passa da primeira para a segunda região, e como consequência o aumento na probabilidade de transmissão se torna uma diminuição.

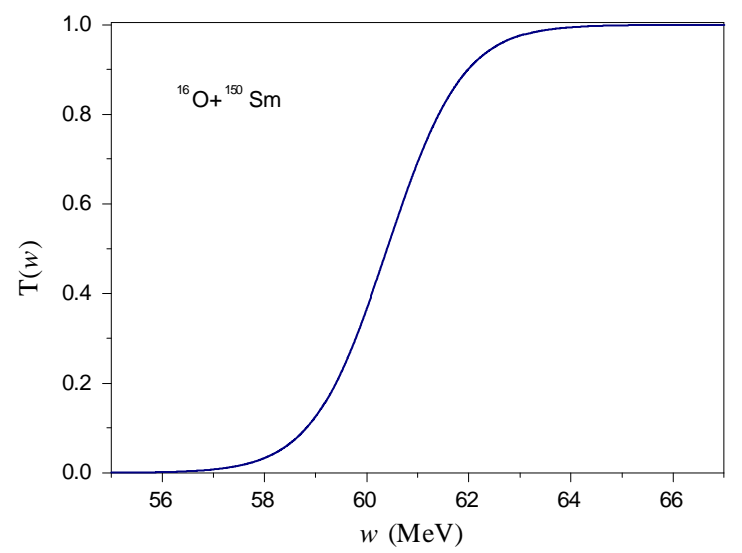

Figura 10: Probabilidade de transmissão versus a função $w(\zeta)$ para o sistema ${ }^{16} \mathrm{O}+{ }^{150} \mathrm{Sm}$, para $l=0$. Na região $0 \leqslant T(w) \leqslant 0.5$, a curva mostra uma dependência convexa do funcional 
probabilidade de tunelamento com relação à função $w(\zeta)$, enquanto que na região classicamente permitida, a curva torna-se côncava.

Essa propriedade geral de convexidade da probabilidade de tunelamento para $T_{l}(w) \leqslant 0.5$ implica um aumento no tunelamento (fusão), com relação a uma comparação com um modelo unidimensional, em conformidade com o que os dados experimentais parecem claramente apontar [45].

No caso da concavidade da curva da probabilidade de transmissão não ser a mesma para todos os possíveis valores de $w$, que é o caso das curvas mostradas acima, a desigualdade de Jensen poderá ser usada se $\left|\omega(\zeta)-\langle\omega(\zeta)\rangle_{\zeta}\right|<K$, onde $K$ é um limite superior para os desvios da distribuição $w(\zeta)$. Em outras palavras, se os desvios de todos os possíveis valores da distribuição $w(\zeta)$ com relação ao valor médio $\langle w(\zeta)\rangle_{\zeta}$ forem suficientemente pequenos de modo que a distribuição $w(\zeta)$ fique totalmente contida em um dos intervalos nos quais a concavidade não muda, então podemos enunciar:

$$
\left\langle T_{0}\left[E, V_{N_{0}}(r)+F\left(R_{l}\right) G(\zeta)\right]\right\rangle_{\zeta} \geq T_{0}\left[E, V_{N_{0}}(r)+F\left(R_{l}\right)\langle G(\zeta)\rangle_{\zeta}\right]
$$

se a distribuição $w(\zeta)$ está contida no intervalo em que o gráfico é convexo, ou

$$
\left\langle T_{0}\left[E, V_{N_{0}}(r)+F\left(R_{l}\right) G(\zeta)\right]\right\rangle_{\zeta} \leq T_{0}\left[E, V_{N_{0}}(r)+F\left(R_{l}\right)\langle G(\zeta)\rangle_{\zeta}\right]
$$

se a distribuição está contida no intervalo em que o gráfico é côncavo. Se a distribuição $w(\zeta)$ for tão dispersa a ponto de não poder ser restrita a um destes intervalos, a desigualdade de Jensen não pode ser utilizada. No entanto, façamos aqui uma ressalva a essa questão. No caso do acoplamento ao meio externo ser representado por um oscilador harmônico, a energia potencial relacionada a esse oscilador poderia assumir infinitos valores, não permitindo então, a princípio, que a distribuição $w(\zeta)$ seja restrita a um intervalo específico. No entanto, interpretando fisicamente a distribuição $w(\zeta)$ como sendo "praticamente"contida numa determinada faixa de energia, podemos proceder a mesma análise acima, de forma aproximada. 


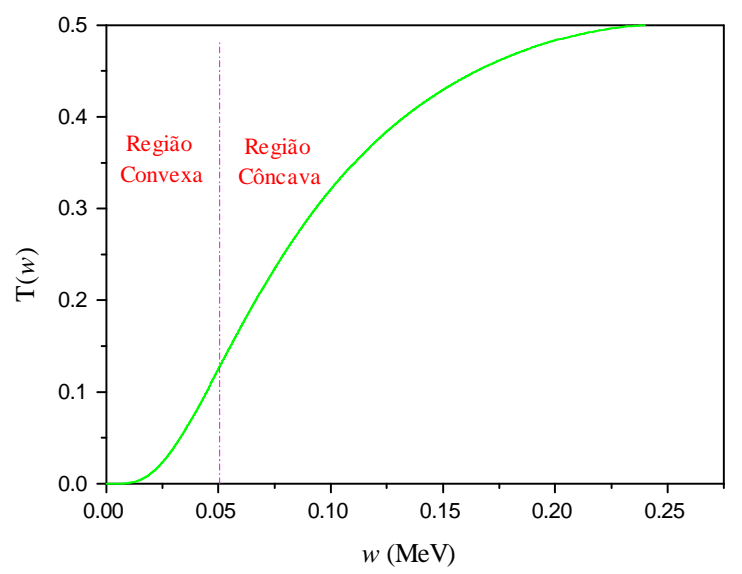

Figura 11: Exemplo de gráfico de probabilidade de tunelamento em função de $w(\zeta)$, onde a concavidade da curva não é constante. Separando o gráfico em duas regiões de concavidades distintas, pode-se aplicar a desigualdade de Jensen, desde que a distribuição $w(\zeta)$ esteja completamente contida em uma delas.

Entretanto, gráficos da probabilidade de transmissão como função de $w(\zeta)$ para sistemas de íons muito leves mostram um comportamento diferente no que concerne à concavidade das curvas, como mostrado nas Figuras (12), (13) e (14). Os gráficos seguintes foram obtidos usando o mesmo método descrito anteriormente i.e. a probabilidade de transmissão foi definida pelas Eqs. (3.0.11), (3.0.12) e pelas Eqs. (3.0.13), (3.0.14); respectivamente para valores de $w$ abaixo e acima do topo da barreira. A barreira de potencial utilizada foi também dada pela Eq. (4.5.14), onde a interação nuclear tem a forma Woods-Saxon. Para tais íons leves, as curvas de $T_{l}$ versus $w(\zeta)$ apresentam três pontos de inflexão ao invés de apenas um, tornando-se côncavas antes $w$ atinja o valor correspondente ao topo da barreira de potencial. Este resultado está em contradição com o resultado geral analítico representado pela Eq. (4.3.11), onde a barreira parabólica foi usada como aproximação para a barreira de potencial real. Isso ocorre possivelmente porque, para íons leves, tal aproximação para a barreira de potencial não parece ser adequada, de modo que uma aproximação mais acurada, que levasse em consideração o caráter extremamente assimétrico da curva de potencial, seria requerido para bem representá-la. Um polinômio de grau igual ao superior a três parece se adequar melhor 
a este propósito, mas o tratamento analítico neste caso torna-se deveras mais complicado. Em verdade, o caráter assimétrico de tal barreira de potencial limita até mesmo a validade da forma analítica para a probabilidade de transmissão representada pelas Eqs. (3.0.11), (3.0.12), que são derivadas, através da teoria de aproximação uniforme, a partir do pressuposto de que a barreira de potencial é localmente parabólica.

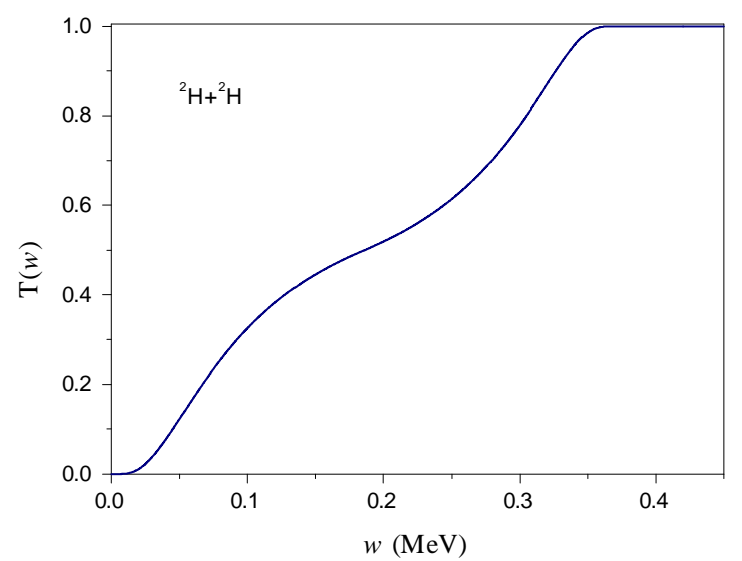

Figura 12: Probabilidade de transmissão versus a função $w(\zeta)$, para o sistema ${ }^{2} H+{ }^{2} H, l=0$. A curva mostra pontos de inflexão extra quando comparada às curvas correspondentes para sistemas mais pesados, o que se deve ao caráter mais assimétrico da curva de interação ion-íon para esses sistemas.

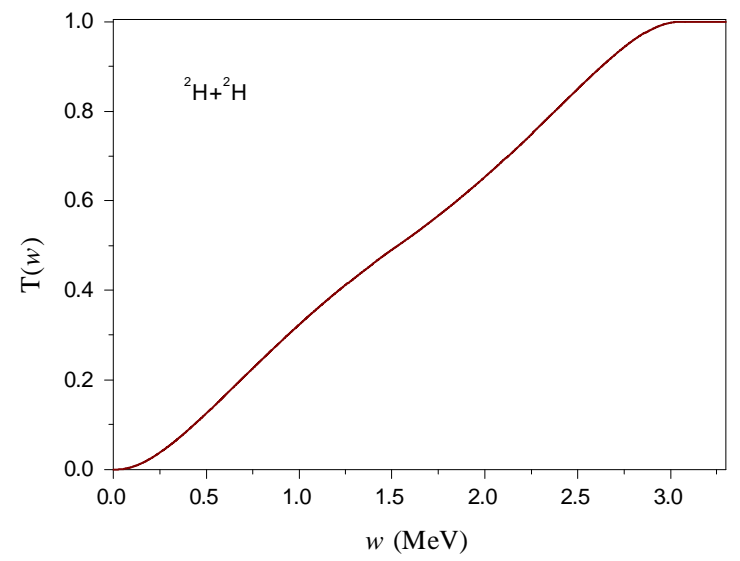


Figura 13: Probabilidade de transmissão versus a função $w(\zeta)$, para o sistema ${ }^{2} H+{ }^{2} H, l=1$. A curva apresenta uma atenuação evidente em sua excentricidade, quando comparada à curva para $l=0$ do mesmo sistema.

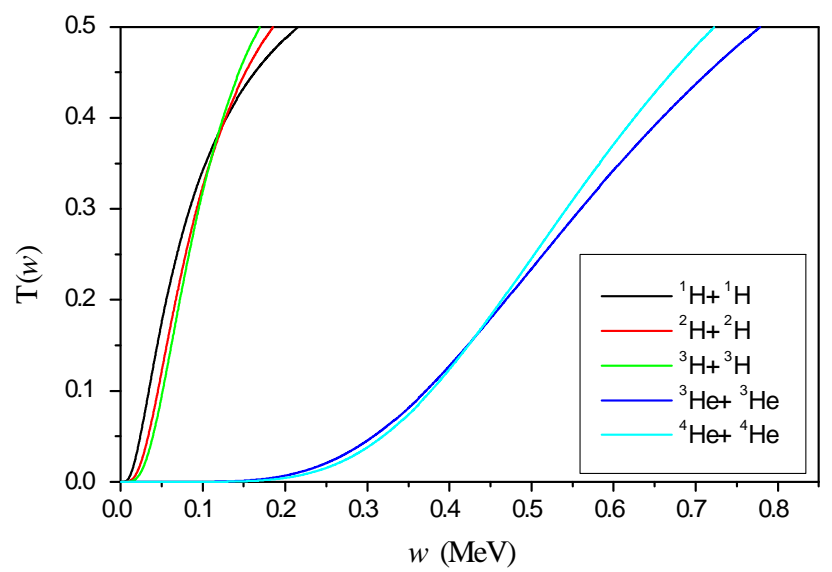

Figura 14: Probabilidade de tunelamento em função de w para diversos pares de ions leves, para a onda parcial $l=0$. Em todas as curvas, é notada uma mudança de concavidade à medida que $w$ cresce.

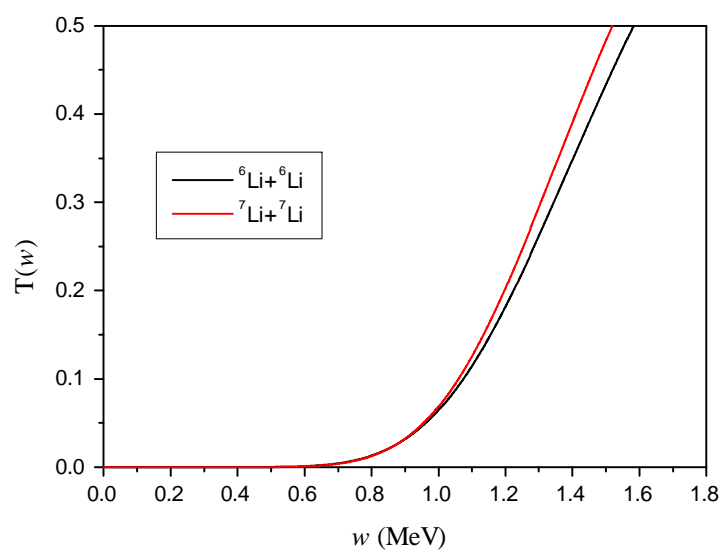

Figura 15: Probabilidade de tunelamento em funcão de $w(\zeta)$ para isótopos do lítio, para a onda parcial $l=0$. 


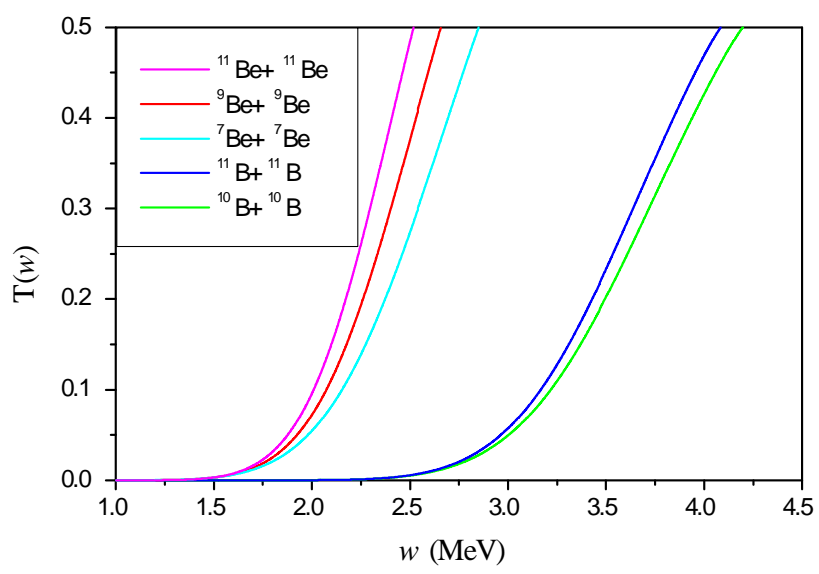

Figura 16: Probabilidade de tunelamento em funcão de $w(\zeta)$ para isótopos do berílio e do boro, para a onda parcial $l=0$.

Estes resultados relacionados a íons leves indicam que a aproximação parabólica para a barreira efetiva de potencial não é adequada para tais casos. A aproximação parabólica é a primeira aproximação cabível do potencial real em uma série polinomial. A barreira de potencial para íons muito leves tem um caráter bastante assimétrico, de modo que um polinômio de grau três ou superior seria requerido para bem representar tal barreira. Para íons pesados, por outro lado, a aproximação parabólica proporciona resultados fisicamente interessantes, e ela foi, de fato, utilizada em muitos trabalhos envolvendo graus de liberdade extra na fusão a energias próximas da altura da barreira [44].

\subsection{Desigualdades Envolvendo um Fator de Forma Variante}

Discutiremos agora o caso mais geral em que o fator de forma, $F(r)$, não é uma constante. Retomando a Eq. (3.0.12):

$$
g\left[E, V_{l}(r)+F(r) G(\zeta)\right]=\sqrt{\frac{8 \mu}{\hbar^{2}}} \int_{r_{1}(l, E)}^{r_{2}(l, E)} d r \sqrt{V_{l}(r)+F(r) G(\zeta)-E}
$$


Suponhamos, primeiramente, que $F(r)<0$ para todo $r$. O caso $F(r)>0$ é análogo. Reescrevemos a Eq. (4.6.1) na forma

$$
g\left[E, V_{l}(r)+F(r) G(\zeta)\right]=\sqrt{\frac{8 \mu}{\hbar^{2}}} \int_{r_{1}(l, E)}^{r_{2}(l, E)} d r \sqrt{-F(r)} \sqrt{\frac{V_{l}(r)-E}{(-F(r))}-G(\zeta)}
$$

Fazemos então uma mudança de medida na integral:

$$
d y=d r \sqrt{-F(r)} \Longrightarrow y(r)=\int_{0}^{r} d r^{\prime} \sqrt{-F(r)}
$$

Definimos a função

$$
s(r) \equiv \int_{0}^{r} d r^{\prime} \sqrt{-F\left(r^{\prime}\right)}
$$

tal que $y(r)=s(r)$. Então, introduzindo a função inversa, escrevemos $r=s^{-1}(y)$, e a Eq. (4.6.1) torna-se

$$
\begin{gathered}
g\left[E, V_{l}(r)+F(r) G(\zeta)\right]=\sqrt{\frac{8 \mu}{\hbar^{2}}} \int_{y_{1}}^{y_{2}} d y \sqrt{\frac{V_{l}\left(s^{-1}(y)\right)-E}{\left(-F\left(s^{-1}(y)\right)\right)}-G(\zeta)} \\
=\sqrt{\frac{8 \mu}{\hbar^{2}}} \int_{y_{1}}^{y_{2}} d y \sqrt{V_{\text {eff }}(y)-E_{\text {eff }}}
\end{gathered}
$$

onde $V_{e f f}(y) \equiv \frac{V_{l}\left(s^{-1}(y)\right)-E}{\left(-F\left(s^{-1}(y)\right)\right)}$ e $E_{e f f} \equiv G(\zeta)$. Neste caso, $E_{\text {eff }}$ varia de $V_{e f f \min }$ a $V_{\text {eff max }}$, onde $V_{e f f \text { min }}$ é definido como sendo o valor mínimo da energia requerido para que a probabilidade de tunelamento seja finita, enquanto que $V_{\text {eff max }}$ é a energia correspondente ao topo da barreira de potencial efetiva.

Os limites de integração na Eq. (4.6.5) podem ser encontrados através da Eq. (4.6.3):

$$
y_{1}=\int_{0}^{r_{1}(l, \zeta)} d r^{\prime} \sqrt{-F\left(r^{\prime}\right)}
$$

$\mathrm{e}$

$$
y_{2}=\int_{0}^{r_{2}(l, \zeta)} d r^{\prime} \sqrt{-F\left(r^{\prime}\right)}
$$

Portanto, a princípio é possível reduzir o problema de determinar a probabilidade de tunelamento em um sistema em que potenciais acoplados são considerados, a um problema em que o potencial efetivo e a energia efetiva serão versões modificadas dos potenciais reais envolvidos. Este 
procedimento pode permitir a aplicação analítica de desigualdades envolvendo probabilidades de tunelamento, as quais podem esclarecer efeitos de graus de liberdade acoplados no cálculo de tais probabilidades, como foi mostrado anteriormente. 


\section{CAPÍtulo 5}

\section{Conclusões}

Consideramos neste trabalho algumas propriedades gerais da probabilidade de tunelamento para sistemas acoplados a um reservoir. Utilizando a desigualdade de Jensen, mostramos que, usando a teoria da aproximação uniforme para a probabilidade de tunelamento, a média da probabilidade de tunelamento é, em geral, maior que a probabilidade de tunelamento calculada com os graus de liberdade relacionados ao reservoir sendo tomados em média. Por outro lado, a probabilidade média de transmissão a energias acima da barreira é, em geral, menor que a probabilidade de transmissão calculada com os graus de liberdade relacionados ao reservoir sendo tomados em média. Esse fato tem uma consequência imediata na fusão de íons pesados a energias próximas da altura da barreira, cujos dados experimentais parecem claramente indicar um aumento do tunelamento devido ao acoplamento com o reservoir (efeito de canais acoplados). Adicionalmente, mostramos que os resultados obtidos por JG [40] podem ser generalizados usando a desigualdade de Jensen. A subjacente dependência matemática da probabilidade de transmissão como função do acoplamento ao reservoir, a saber, o fato da probabilidade de tunelamento ser em geral um funcional convexo do hamiltoniano de acoplamento na região classicamente proibida, e por outro lado um funcional côncavo do acoplamento na região classicamente permitida evidenciam a eficácia da aplicação da desigualdade de Jensen neste campo de pesquisa, com o intuito de comparar duas formas diferentes de probabilidades de reação, ambas de interesse físico. 
As desigualdades obtidas neste trabalho dão lastro à idéia de que o modelo de penetração em barreira unidimensional incorre em desvios intrínsecos dos valores esperados para coeficientes de transmissão, ainda que seus parâmetros sejam ajustados da melhor maneira possível de modo a representar com fidedignidade o fenômeno físico de que trata.

As desigualdades (4.3.11) e (4.3.13), obtidas somente através da aplicação da desigualdade de Jensen a uma forma analítica do coeficiente de transmissão, no qual consideramos um acoplamento linear ao reservoir e uma barreira de potencial aproximadamente parabólica, tem uma correspondência direta com um resultado empiricamente bem conhecido, obtido através de cálculos numéricos para diferentes modelagens; a saber, o acoplamento linear a um oscilador aumenta a probabilidade de tunelamento a energias abaixo da barreira de potencial original na ausência de acoplamento, enquanto diminui a probabilidade de transmissão a energias acima da barreira. Com efeito, as desigualdades (4.3.11) e (4.3.13) levam exatamente a essa regra no caso especial em que $\left\langle H_{\text {int }}\left(R_{l}, \zeta\right)\right\rangle_{\zeta}=0$

Nossos resultados para íons leves, mostrados nas Figuras (12), (13), (14) indicam que seria contraproducente o uso da desigualdade de Jensen aplicada à aproximação uniforme para a probabilidade de tunelamento de tais sistemas, visto que a desigualdade é revertida várias vezes à medida que a energia efetiva varia. O comportamento peculiar apresentado pelas curvas de probabilidade de tunelamento para íons leves sugere que para tais sistemas um tratamento semiclássico mais acurado de tunelamento através de barreiras de potencial assimétricas se faz necessário [23] para determinar os efeitos da presença do reservoir.

Discutindo os resultados de [6-8] à luz da desigualdade de Jensen, é-se inclinado a assumir que a probabilidade de tunelamento considerada por estes autores é um funcional côncavo do acoplamento ao reservoir, visto que seus resultados últimos parecem indicar uma atenuação da barreira de penetração devido a esse acoplamento. O caso de fusão de deutérios em meios metálicos pode ser discutido da mesma maneira, de modo que neste caso a probabilidade de tunelamento considerada parece ser um funcional convexo do acoplamento ao reservoir, já que a probabilidade de tunela- 
mento é aumentada (fisicamente, pode-se atribuir isto à blindagem eletrônica, que resulta numa atenuação da barreira Coulombiana). 


\section{CAPÍtulo 6}

\section{APÊNDICE}

Neste apêndice nós discutimos as aproximações que fizemos na Eq. (3.0.14), que nós aqui repetimos:

$$
g_{l}^{a}\left[E, V_{l}(r)+H_{\text {int }}\left(R_{l}, \zeta\right)\right]=\sqrt{\frac{8 \mu}{\hbar^{2}}} \int_{z_{1}(l, \zeta)}^{z_{2}(l, \zeta)} d r \sqrt{V_{l}(r)+H_{\text {int }}\left(R_{l}, \zeta\right)-E}
$$

para fazer o cálculo númérico da ação $g_{l}^{a}$, utilizado nos gráficos mostrados nas Figuras (9), (10), (12), (13), em que a probabilidade de transmissão é calculada abaixo e acima da barreira.

Quando o potencial $V_{l}(r)$ tem uma forma anlítica complicada tal qual a função que representa o potencial de estamos utilizando (Eq. (4.5.5)), a tarefa de encontrar as raízes complexas da equação $V_{l}(r)+H_{\text {int }}\left(R_{l}, \zeta\right)-E=0$ se torna inexequível. Ademais, analiticamente mais de dois pontos de retorno são provavelmente esperados.

S. C. Miller e R. H. Good, discutindo o problema da determinação do coeficiente de transmissão para energias acima da barreira de tunelamento, utilizando seu método de funções de comparação, em seu artigo intitulado "A WKB-Type Approximation to the Shrödinger equation" [23], explicitamente dizem que restringiriam a discussão ao caso no qual "os dois pontos de retorno reais para energias abaixo do pico da barreira levam inambiguamente a dois pontos de retorno complexos acima do pico da barreira". Um procedimento análogo foi também seguido por Kemble [22]. Nesse contexto, a aproximação parabólica surge como a escolha mais óbvia que poderia fornecer uma 
relação biunívoca entre os pontos de retorno reais para energias abaixo da barreira e os pontos de retorno complexos para energias acima da barreira.

Para realizar uma "abordagem por aproximação parabólica"na determinação dos pontos de retorno sem efetivamente alterar o potencial real dentro da raíz quadrada na integral contida na Eq. (3.0.14), notemos primeiramente que para uma barreira parabólica, a transmissão sobre a barreira com energia $E$, maior que a altura da barreira $V_{0}$ por uma diferença $\Delta\left(\Delta=\left|E-V_{0}\right|\right)$ é análoga, com relação à determinação dos pontos de retorno, ao tunelamento através da barreira com energia $E^{\prime}$, com $E^{\prime}=V_{0}-\Delta$. Estas duas situações estão ilustradas abaixo nas Figuras (17) e (18).

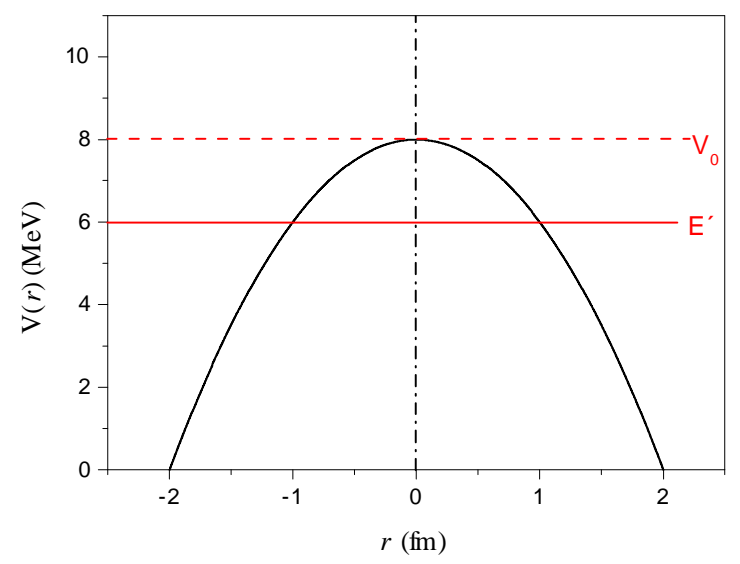

Figura 17: Esquema representando o tunelamento através da barreira de potencial. Aqui, $V_{0}=8$ $M e V$ e $\Delta=2 M e V$.

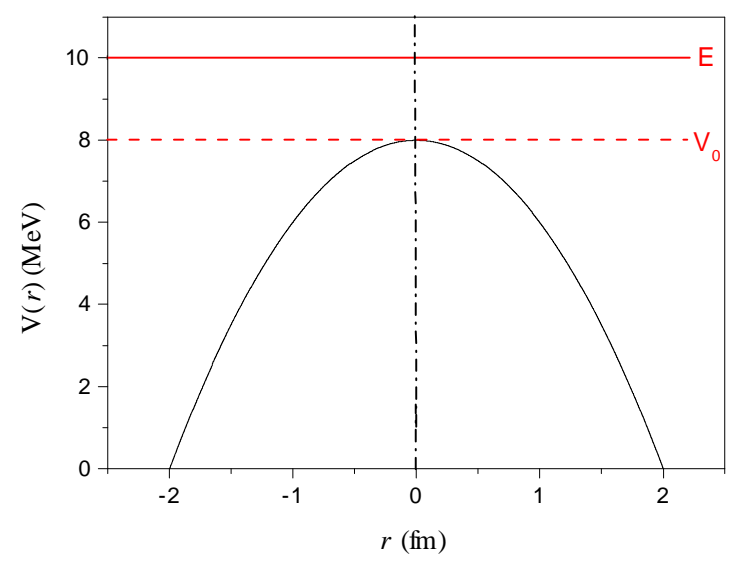


Figura 18: Esquema representando a transmissão acima da barreira de potencial. Assim como na figura anterior, $V_{0}=8 \mathrm{MeV}$ e $\Delta=2 \mathrm{MeV}$.

Dada uma barreira parabólica do tipo $V(x)=V_{0}-k r^{2}$, e uma dada energia $E, E>V_{0}$, calculemos as raízes de $V_{0}-k r^{2}-E=0$ :

$$
V_{0}-k r^{2}-E=0 \Rightarrow r= \pm i \sqrt{\frac{\left|V_{0}-E\right|}{k}}= \pm i \sqrt{\frac{E-V_{0}}{k}} \equiv \pm i x
$$

Este procediemnto é equivalente à determinação dos pontos de retorno complexos relacionados à Fig. (18). Agora, dada uma barreira parabólica do tipo $V(x)=V_{0}-k r^{2}$ e uma dada energia $E^{\prime}=V_{0}-\left(E-V_{0}\right), E>V_{0}$, calculemos as raízes da equação $V_{0}-k r^{2}-\left(V_{0}-\left(E-V_{0}\right)\right)=0$ :

$$
V_{0}-k r^{2}-\left(V_{0}-\left(E-V_{0}\right)\right)=0 \Rightarrow r= \pm \sqrt{\frac{E-V_{0}}{k}} \equiv \pm x
$$

Este procedimento é equivalente à determinação dos pontos de retorno reais relacionados à Fig. (17). Colocando em palavras, temos que a determinação dos pontos de retorno complexos para o problema de transmissão acima de barreira parabólica, com energia $E=V_{0}+\Delta$ é análoga à determinação dos pontos de retorno reais para o problema de tunelamento através de uma barreira de potencial parabólica, com energia $E^{\prime}=V_{0}-\Delta$, em que $\Delta=\left|E-V_{0}\right|$. E deve ser observado que este resultado é inerente à assumição de que a barreira de potencial é parabólica. Estes argumentam sustentam a seguinte forma de aproximação para o cálculo de $g_{l}^{a}$ :

$$
g_{l}^{a}\left[E, V_{l}(r)+H_{\text {int }}\left(R_{l}, \zeta\right)\right] \equiv \sqrt{\frac{8 \mu}{\hbar^{2}}} \int_{r_{1}(l, \zeta)}^{r_{2}(l, \zeta)} d r \sqrt{E-\left(V_{l}(r)+H_{\text {int }}\left(R_{l}, \zeta\right)\right)}
$$

em que $E>V_{l}(r)+H_{\text {int }}\left(R_{l}, \zeta\right)$ e $r_{1}(l, \zeta), r_{2}(l, \zeta)$ são as raízes (reais) da equação $E-\left(V_{l}(r)+H_{\text {int }}\left(R_{l}, \zeta\right)\right)=$ 0. Note-se que $V_{l}(r)$ na Eq. (6.0.1) ainda representa o potencial dado pela Eq. (4.5.5) e não uma parábola. Portanto, nós fizemos uma "aproximação parabólica"sobre o potencial com o intuito de achar os correspondentes pontos de retorno, mas mantivemos o potencial original para o cálclo numérico da ação $g_{l}^{a}$. 


\section{Referências Bibliográficas}

[1] R. G. Stokstad et al., Phys. Rev. Lett., 41, 465 (1978).

[2] R. G. Stokstad et al., Z. Phys. A 295, 269 (1980).

[3] M. Beckerman et al., Phys. Rev. C, 23, 1581 (1981).

[4] M. Beckerman et al. Phys. Rev. C, 25, 837 (1982).

[5] W. Reisdorf et al., Phys. Rev. Lett., 49, 1811 (1982).

[6] A. O. Caldeira e A. J. Leggett, Phys. Rev. Lett., 46, 211 (1981).

[7] A. O. Caldeira e A. J. Leggett, Phys. Rev. Lett., 48, 1571 (1982).

[8] A. O. Caldeira e A. J. Leggett, Ann. Phys. (N. Y.), 149, 374 (1983). 
[9] R. P. Feynman e F. L. Vernon Jr., Ann. Phys. (N. Y.), 24, 118 (1963).

[10] R. P. Feynman e A. Hibbs, "Quantum Mechanics and Path Integrals", McGraw-Hill, New York, (1965).

[11] P. M. Jacob e U. Smilansky, Phys. Lett. B, 127, 313 (1983).

[12] D. M. Brink e U. Smilansky, Nucl. Phys. A, 405, 301 (1983).

[13] D. M. Brink, M. C. Nemes e D. Vautherin, Ann. Phys. (N. Y.), 147, 171 (1983).

[14] M. S. Hussein, Phys. Rev. C, 30, 1962 (1984).

[15] G. R. Satchler, M. N. Nagarajan, J. S. Lilley e I. J. Thompson, Ann. Phys., 178, 110 (1987).

[16] C. H. Dasso, S. Landowne e A. Winther, Nucl. Phys. A, 405, 381 (1983);ibid 443, 495 (1985).

[17] C. H. Dasso e S. Landowne, Comput. Phys. Commun, 46, 187 (1987); J. Fernández-Niello, C. H. Dasso e S. Landowne, ibid, 54, 409 (1989) [CCFUS]

[18] K. Hagino, N. Rowley e A. T. Kruppa, Comput. Phys. Commun., 123, 143 (1999) [CCFULL].

[19] A. R. Plastino, A. Plastino e G. H. Miller, Am. J. Phys., 65 (11), 1102 (1997) 
[20] R. Peierls, Phys. Rev. 54, 918 (1938).

[21] J. Ruel e M. Ayres, Trends in Ecology and Evolution, vol 14, num 9, (1999)

[22] E. C. Kemble, Phys. Rev., 48, 549 (1935).

[23] S. C. Miller e R. H. Good, Phys. Rev. 91, 174 (1953).

[24] D. L. Hill e J. A. Wheeler, Phys. Rev., 89, 1102 (1953).

[25] G. Gamow, Z. Phys., 51, 204 ( 1928).

[26] H. J. Rose e G. A. Jones, Nature, 307, 245 (1984).

[27] S. Landowne e C. H. Dasso, Phys. Rev. C, 33, 387 (1985).

[28] C. Y. Wong, Phys. Rev. Lett., 31, 766 (1973).

[29] R. E. Langer, Phys. Rev., 51, 669 (1937).

[30] N. T. Maitra e E. Heller, Phys. Rev. A 4763 (1996).

[31] A. B. Balantekin e N. Takigawa, Ann. Phys. (N. Y.), 160, 441 (1985). 
[32] B. Müller e N. Takigawa, Ann. Phys. (N. Y.), 163 (1987).

[33] A. B. Balantekin e N. Takigawa, Rev. Mod. Phys., 70, 77 (1998).

[34] D. M. Brink e N. Takigawa, Nucl. Phys. A279, 159 (1977).

[35] See, e.g., L. F. Canto, P. R. S. Gomes, R. Donangelo e M. S. Hussein, Physics Reports, 424, 1 (2006).

[36] C. L. Jiang, et al. Phys. Rev. Lett. 89, 052701 (2002).

[37] C. L. Jiang, et al. Phys. Rev. C 73, 014613 (2006).

[38] E. H. Lieb e M. Loss, "Analysis", American Mathematical Society, 1997.

[39] C. Chicone, Journal of Differential Equations, 69, 310 (1987).

[40] R. C. Johnson e C. J. Goebel, Phys. Rev. C, 62, 027603 (2000).

[41] J. S. Al-Khalili e J. A. Tostevin, Phys. Rev. Lett., 76, 3903 (1996).

[42] F. Calogero e F. Leyvraz, J. Math. Phys. 47, Issue 4 (2006).

[43] O. A. Chalykh e A. P. Veselov, Journal of Nonlinear Mathematical Physics 12 supplement 1, 179 (2005). 
[44] G. P. A. Nobre, L. C. Chamon, B. V. Carlson, I. J. Thompson e L.R. Gasques, Nucl. Phys. A, 786, 90 (2007).

[45] M. Dasgupta, D. Hinde, N. Rowley e A. Stefanini, Ann. Rev. Nucl. Part. Sci. 48, 401 (1998).

[46] L. C. Chamon, D. Pereira, M. S. Hussein, M. A. C. Ribeiro, e D. Galetti, Phys. Rev. Lett. 79, 5218 (1997).

[47] L. C. Chamon et al., Phys. Rev.C 66, 014610 (2002). 